\title{
A SHARP CONDITION FOR SCATTERING OF THE RADIAL 3D CUBIC NONLINEAR SCHRÖDINGER EQUATION
}

\author{
JUSTIN HOLMER AND SVETLANA ROUDENKO
}

\begin{abstract}
We consider the problem of identifying sharp criteria under which radial $H^{1}$ (finite energy) solutions to the focusing $3 \mathrm{~d}$ cubic nonlinear Schrödinger equation (NLS) $i \partial_{t} u+\Delta u+|u|^{2} u=0$ scatter, i.e. approach the solution to a linear Schrödinger equation as $t \rightarrow \pm \infty$. The criteria is expressed in terms of the scale-invariant quantities $\left\|u_{0}\right\|_{L^{2}}\left\|\nabla u_{0}\right\|_{L^{2}}$ and $M[u] E[u]$, where $u_{0}$ denotes the initial data, and $M[u]$ and $E[u]$ denote the (conserved in time) mass and energy of the corresponding solution $u(t)$. The focusing NLS possesses a soliton solution $e^{i t} Q(x)$, where $Q$ is the ground-state solution to a nonlinear elliptic equation, and we prove that if $M[u] E[u]<M[Q] E[Q]$ and $\left\|u_{0}\right\|_{L^{2}}\left\|\nabla u_{0}\right\|_{L^{2}}<\|Q\|_{L^{2}}\|\nabla Q\|_{L^{2}}$, then the solution $u(t)$ is globally well-posed and scatters. This condition is sharp in the sense that the soliton solution $e^{i t} Q(x)$, for which equality in these conditions is obtained, is global but does not scatter. We further show that if $M[u] E[u]<M[Q] E[Q]$ and $\left\|u_{0}\right\|_{L^{2}}\left\|\nabla u_{0}\right\|_{L^{2}}>\|Q\|_{L^{2}}\|\nabla Q\|_{L^{2}}$, then the solution blows-up in finite time. The technique employed is parallel to that employed by Kenig-Merle [16] in their study of the energy-critical NLS.
\end{abstract}

\section{INTRODUCTION}

Consider the cubic focusing nonlinear Schrödinger (NLS) equation on $\mathbb{R}^{3}$ :

$$
i \partial_{t} u+\Delta u+|u|^{2} u=0,
$$

where $u=u(x, t)$ is complex-valued and $(x, t) \in \mathbb{R}^{3} \times \mathbb{R}$. The initial-value problem posed with initial-data $u(x, 0)=u_{0}(x)$ is locally well-posed in $H^{1}$ (see Ginibre-Velo [9]; standard reference texts are Cazenave [3, Linares-Ponce [20], and Tao [29]). Such solutions, during their lifespan $\left[0, T^{*}\right)$ (where $T^{*}=+\infty$ or $T^{*}<+\infty$ ), satisfy mass conservation $M[u](t)=M\left[u_{0}\right]$, where

$$
M[u](t)=\int|u(x, t)|^{2} d x
$$

and energy conservation $E[u](t)=E\left[u_{0}\right]$, where

$$
E[u](t)=\frac{1}{2} \int|\nabla u(x, t)|^{2} d x-\frac{1}{4} \int|u(x, t)|^{4} d x
$$

(and we thus henceforth denote these quantities $M[u]$ and $E[u]$ respectively, with no reference to the time $t$ ). 
The equation also has several invariances, among them (in each of the following cases, $\tilde{u}$ is a solution to (1.1) if and only if $u$ is a solution to (1.1)):

- Spatial translation. For a fixed $x_{0} \in \mathbb{R}^{3}$, let $\tilde{u}(x, t)=u\left(x+x_{0}, t\right)$.

- Scaling. For a fixed $\lambda \in(0,+\infty)$, let $\tilde{u}(x, t)=\lambda u\left(\lambda x, \lambda^{2} t\right)$.

- Galilean phase shift. For a fixed $\xi_{0} \in \mathbb{R}^{3}$, let $\tilde{u}(x, t)=e^{i x \xi_{0}} e^{-i t \xi_{0}^{2}} u\left(x-2 \xi_{0} t, t\right)$.

The scale-invariant Sobolev norm is $\dot{H}^{1 / 2}$, although we find it more useful, as described below, to focus on the scale invariant quantities $\|u(t)\|_{L^{2}}\|\nabla u(t)\|_{L^{2}}$ and $M[u] E[u]$. The Galilean invariance leaves only the $L^{2}$ norm invariant, while translation leaves all Sobolev norms invariant. We note that these two symmetries do not preserve radiality, while the scaling symmetry does.

The nonlinear elliptic equation

$$
-Q+\Delta Q+|Q|^{2} Q=0, \quad Q=Q(x), \quad x \in \mathbb{R}^{3},
$$

has an infinite number of solutions in $H^{1}$. Among these there is exactly one solution of minimal mass 1 , called the ground-state solution, and it is positive (real-valued), radial, smooth, and exponentially decaying (see Appendix B of Tao's text [29] for exposition). We henceforth denote by $Q$ this ground-state solution. If we let $u(x, t)=e^{i t} Q(x)$, then $u$ is a solution to (1.1), and is called the standard soliton. A whole family of soliton solutions to (1.1) can be built from the standard soliton via the invariances of the NLS equation (1.1):

$$
u(x, t)=e^{i t} e^{i x \cdot \xi_{0}} e^{-i t\left|\xi_{0}\right|^{2}} \lambda u\left(\lambda\left(x-\left(x_{0}+2 \xi_{0} t\right)\right), \lambda^{2} t\right) .
$$

The standard soliton has the property that the quantities $\left\|u_{0}\right\|_{L^{2}}\left\|\nabla u_{0}\right\|_{L^{2}}$ and $M[u] E[u]$ are minimal among all solitons (1.3). Indeed, these quantities are independent of translation and scaling, and the introduction of a Galilean phase shift only increases their values. Since solutions to the linear Schrödinger equation completely disperse (spread out, and shrink in a variety of spatial norms) as $t \rightarrow \pm \infty$, the soliton solutions by their definition do not scatter (approach a solution of the linear Schrödinger equation). Indeed, soliton solutions represent a perfect balance between the focusing forces of the nonlinearity and the dispersive forces of the linear component.

The basic line of thought in the subject, motivated by heuristics (Soffer [24]), rigorous partial results (Tao [27, 28]), numerical simulation (Sulem-Sulem [26]), and analogy with the completely integrable one-dimensional case, is that a solution of (1.1) either completely disperses as $t \rightarrow \infty$ (linear effects dominate), blows-up in finite time (nonlinear effects dominate) or the solution resolves into a sum of solitons

\footnotetext{
${ }^{1}$ In view of the connection between solutions $Q$ to (1.2) and solutions $u(t)=e^{i t} Q$ to (1.1), and the fact that $\|u(t)\|_{L^{2}}\|\nabla u(t)\|_{L^{2}}$ is a scale invariant quantity for solutions $u(t)$ to (1.1), it might be more natural to classify the family of solutions $Q$ to (1.2) in terms of the quantity $\|Q\|_{L^{2}}\|\nabla Q\|_{L^{2}}$ rather than the mass. However, any solution $Q$ to (1.2) must satisfy the Pohozhaev identity $\|Q\|_{L^{2}}\|\nabla Q\|_{L^{2}}=\sqrt{3}\|Q\|_{L^{2}}^{2}$, and thus the two classifications are equivalent.
} 
propagating in different directions or at different speeds plus dispersive radiation as $t \rightarrow \infty$ (nonlinear effects and linear effects balance). Since the smallest value of $\left\|u_{0}\right\|_{L^{2}}\left\|\nabla u_{0}\right\|_{L^{2}}$ among all soliton solutions is $\|Q\|_{L^{2}}\|\nabla Q\|_{L^{2}}$, it seems reasonable to conjecture, even for nonradial data, that if $\left\|u_{0}\right\|_{L^{2}}\left\|\nabla u_{0}\right\|_{L^{2}}<\|Q\|_{L^{2}}\|\nabla Q\|_{L^{2}}$, then the solution scatters provided we can rule out blow-up. Ruling out blow-up in this situation is straightforward provided $M[u] E[u]<M[Q] E[Q]$ using the conservation of mass and energy and a result of $\mathrm{M}$. Weinstein stating that an appropriate GagliardoNirenberg inequality is optimized at $Q$. The main result of this paper is the resolution of this conjecture under the assumption of radial data, which appears below as Theorem 1.1(1) (b).

Theorem 1.1. Let $u_{0} \in H^{1}$ be radial and let $u$ be the corresponding solution to (1.1) in $H^{1}$ with maximal forward time interval of existence $[0, T)$. Suppose $M[u] E[u]<$ $M[Q] E[Q]$.

(1) If $\left\|u_{0}\right\|_{L^{2}}\left\|\nabla u_{0}\right\|_{L^{2}}<\|Q\|_{L^{2}}\|\nabla Q\|_{L^{2}}$, then

(a) $T=+\infty$ (the solution is globally well-posed in $H^{1}$ ), and

(b) $u$ scatters in $H^{1}$. This means that there exists $\phi_{+} \in H^{1}$ such that

$$
\lim _{t \rightarrow+\infty}\left\|u(t)-e^{i t \Delta} \phi_{+}\right\|_{H^{1}}=0 .
$$

(2) If $\left\|u_{0}\right\|_{L^{2}}\left\|\nabla u_{0}\right\|_{L^{2}}>\|Q\|_{L^{2}}\|\nabla Q\|_{L^{2}}$, then $T<+\infty$ (the solution blows-up in finite time).

It is straightforward to establish as a corollary the same result for negative times: take the complex conjugate of the equation and replace $t$ by $-t$. Since the hypotheses in Theorem 1.1 (11) (2) apply to $u_{0}$ if and only if they apply to $\bar{u}_{0}$, we obtain that the hypotheses of (11) imply that $u$ scatters both as $t \rightarrow+\infty$ and $t \rightarrow-\infty$ and the hypotheses of (2) imply that $u$ blows-up both in finite positive time and in finite negative time. An interesting open question is whether or not there exist solutions $u$ with $M[u] E[u] \geq M[Q] E[Q]$ that exhibit different behavior in the positive and negative directions.

The proof of Theorem 1.1(1) (b) is based upon ideas in Kenig-Merle [16], who proved an analogous statement for the energy-critical NLS.

The key dynamical quantity in the proof of Theorem 1.1 is a localized variance $\|x u(t)\|_{L^{2}(|x| \leq R)}$. The virial identity states that if $\left\|x u_{0}\right\|_{L^{2}}<\infty$, then $u$ satisfies

$$
\partial_{t}^{2} \int|x|^{2}|u(x, t)|^{2} d x=24 E[u]-4\|\nabla u(t)\|_{L_{x}^{2}}^{2} .
$$

We use a localized version of this identity in both the proof of Theorem 1.1(2) and the rigidity lemma (see $\S[6)$ giving Theorem 1.1(1) (b). On a heuristic level (keeping in mind that $\|u(t)\|_{L^{2}}$ is conserved), under the hypotheses of Theorem 1.1(1), the right side of (1.4) is strictly positive, which pushes the variance $\|x u(t)\|_{L^{2}}$ to $+\infty$ as 
$t \rightarrow+\infty$, which says roughly that the mass of $u$ is being redistributed to large radii, meaning that it "disperses", and we expect the effect of the nonlinearity to diminish and scattering to occur. On the other hand, under the hypotheses of Theorem 1.1(2), the right side of (1.4) is strictly negative, which pushes the variance $\|x u(t)\|_{L^{2}}$ to 0 in finite time, meaning that all the mass of $u$ concentrates at the origin and we expect blow-up. We do not use (1.4) directly, however, for two reasons. First, it requires the additional hypothesis that the initial data has finite variance-an assumption we would like to avoid. Secondly, in the case of the scattering argument, we don't see a method for proving scattering given only the strict convexity (in time) of the variance and its divergence to $+\infty$, although it is heuristically consistent with scattering. The problem is that large variance can be produced by a very small amount of mass moving to very, very large radii, while still leaving a significant amount of mass at small radii. Therefore, to prove the scattering claim in Theorem 1.1(1)(b), we instead use a localized virial identity, as Kenig-Merle [16] did, involving a localized variance. If a very small amount of mass moved to very, very large radii, it would not affect the localized variance dramatically.

For the $3 \mathrm{~d}$ cubic defocusing NLS

$$
i \partial_{t} u+\Delta u-|u|^{2} u=0
$$

scattering has been established for all $H^{1}$ solutions (regardless of "size") even for nonradial data by Ginibre-Velo [10] using a Morawetz inequality. This proof was simplified by Colliander-Keel-Staffilani-Takaoka-Tao [4] using a new interaction Morawetz inequality they discovered. These Morawetz estimates, however, are not positive definite for solutions to the focusing equation (1.1), and thus, cannot be applied directly to our problem. It remains open whether or not one could prove suitable bounds on the nonpositive terms to recover the results of this paper.

For (1.1), Tao [27] proved a few results in the direction of the soliton resolution conjecture, assuming the solution is radial and global (has globally bounded $H^{1}$ norm). It is shown that for large data, radial solutions asymptotically split into (i) a (smooth) function localized near the origin (which is either zero or has a non-zero mass and energy and obeys an asymptotic Pohozhaev identity), (ii) a radiation term evolving by the linear Schrödinger flow, and (iii) an error term (approaching zero in the $\dot{H}^{1}$ norm). Further results for mass supercritical, energy subcritical NLS equations in higher dimensions $(N \geq 5)$ were established by Tao in [28].

The equation (1.1) frequently arises, often in more complex forms, as a model equation in physics. In 2d, it appears as a model in nonlinear optics - see Fibich [7] for a review. When coupled with a nonlinear wave equation, it arises as the Zakharov system [32] in plasma physics. According to [18] p.7, in the mass supercritical case "the most important partial case $p=3, d=3$ corresponds to the subsonic collapse 
of Langmuir waves in plasma". Furthermore, (1.1) arises as a model for the BoseEinstein condensate (BEC) in condensed matter physics. There, it appears as the Gross-Pitaevskii (GP) equation (in 1d, 2d, and 3d), which is (1.1) with a (real) potential $V=V(x)$ :

$$
i \partial_{t} u+\Delta u-V u+a|u|^{2} u=0
$$

It is derived by mean-field theory approximation (see Schlein [25]), and $|u(x, t)|^{2}$ represents the density of the condensate at time $t$ and position $x$. The coefficient $a$ in the nonlinearity is governed by a quantity called the $s$-scattering length. Some elements used in recent experiments $\left({ }^{7} \mathrm{Li},{ }^{85} \mathrm{Rb},{ }^{133} \mathrm{Cs}\right)$ posses a negative $s$-scattering length in the ground state and are modeled by (1.5) with $a<0 . V(x)$ is an external trapping potential imposed by a system of laser beams and is typically taken to be harmonic $V(x)=\beta|x|^{2}$. These "unstable BECs" (where $a<0$ ) have been investigated experimentally recently (see the JILA experiments [6]) and a number of theoretical predictions have been confirmed, including the observation of "collapse events" (corresponding to blow-up of solutions to (1.5)). A few articles have appeared (for example [1]) in the physics literature discussing the critical number of atoms required to initiate collapse. The "critical number of atoms" corresponds to "threshold mass $M[u]$ " in our terminology, and connects well with the mathematical investigations in this paper.

The format of this paper is as follows. In \$2, we give a review of the Strichartz estimates, the small data theory, and the long-time perturbation theory. We review properties of the ground state profile $Q$ in $\$ 3$ and recall its connection to the sharp Gagliardo-Nirenberg estimate of M. Weinstein [31]. In \&4, we introduce the local virial identity and prove Theorem 1.1 except for the scattering claim in part (1) (b). In \$5 6 , we prove Theorem 1.1(1) (b). This is done in two stages, assuming that the threshold for scattering is strictly below the one claimed. First, in $\$ 5$, we construct a solution $u_{\mathrm{c}}$ (a "critical element") that stands exactly at the boundary between scattering and nonscattering. This is done using a profile decomposition lemma in $\dot{H}^{1 / 2}$, obtained by extending the $\dot{H}^{1}$ methods of Keraani [14]. We then show that time slices of $u_{\mathrm{c}}(t)$, as a collection of functions in $H^{1}$, form a precompact set in $H^{1}$ (and thus $u_{\mathrm{c}}$ has something in common with the soliton $\left.e^{i t} Q(x)\right)$. This enables us to prove that $u_{\mathrm{c}}$ remains localized uniformly in time. In $\sqrt[6]{6}$, this localization is shown to give a strict convexity (in time) of a localized variance which leads to a contradiction with the conservation of mass at large times. In \$7, we explain how Theorem 4.2 should carry over to more general nonlinearities and general dimensions (mass supercritical and energy subcritical cases) of NLS equations.

Acknowledgement. J.H. is partially supported by an NSF postdoctoral fellowship. S.R. would like to thank Mary and Frosty Waitz for their great hospitality 
during her visits to Berkeley. We both thank Guixiang $\mathrm{Xu}$ for pointing out a few misprints and the referee for helpful suggestions.

\section{LocAl THEORY AND StRICHARTZ ESTimates}

We begin by recalling the relevant Strichartz estimates (e.g., see Cazenave [3], Keel-Tao [15]). We say that $(q, r)$ is $\dot{H}^{s}$ Strichartz admissible (in 3d) if

$$
\frac{2}{q}+\frac{3}{r}=\frac{3}{2}-s
$$

Let

$$
\|u\|_{S\left(L^{2}\right)}=\sup _{\substack{(q, r) L^{2} \text { admissible } \\ 2 \leq r \leq 6,2 \leq q \leq \infty}}\|u\|_{L_{t}^{q} L_{x}^{r}} .
$$

In particular, we are interested in $(q, r)$ equal to $\left(\frac{10}{3}, \frac{10}{3}\right)$ and $(\infty, 2)$. Define 2

$$
\|u\|_{S\left(\dot{H}^{1 / 2}\right)}=\sup _{\substack{(q, r) \dot{H}^{1 / 2} \text { admissible } \\ 3 \leq r \leq 6^{-}, 4^{+} \leq q \leq \infty}}\|u\|_{L_{t}^{q} L_{x}^{r}},
$$

where $6^{-}$is an arbitrarily preselected and fixed number $<6$; similarly for $4^{+}$. We will, in particular, use $(q, r)$ equal to $(5,5),\left(20, \frac{10}{3}\right)$, and $(\infty, 3)$. Now we consider dual Strichartz norms. Let

$$
\|u\|_{S^{\prime}\left(L^{2}\right)}=\inf _{\substack{(q, r) L^{2} \text { admissible } \\ 2 \leq q \leq \infty, 2 \leq r \leq 6}}\|u\|_{L_{t}^{q^{\prime}} L_{x}^{r^{\prime}}}
$$

where $\left(q^{\prime}, r^{\prime}\right)$ is the Hölder dual to $(q, r)$. Also define

$$
\|u\|_{S^{\prime}\left(\dot{H}^{-1 / 2}\right)}=\inf _{\substack{(q, r) \dot{H}^{-1 / 2} \text { admissible } \\ \frac{4}{3}^{+} \leq q \leq 2^{-}, 3^{+} \leq r \leq 6^{-}}}\|u\|_{L_{t}^{q^{\prime}} L_{x}^{r^{\prime}}} .
$$

The Strichartz estimates are

$$
\left\|e^{i t \Delta} \phi\right\|_{S\left(L^{2}\right)} \leq c\|\phi\|_{L^{2}}
$$

and

$$
\left\|\int_{0}^{t} e^{i\left(t-t^{\prime}\right) \Delta} f\left(\cdot, t^{\prime}\right) d t^{\prime}\right\|_{S\left(L^{2}\right)} \leq c\|f\|_{S^{\prime}\left(L^{2}\right)}
$$

By combining Sobolev embedding with the Strichartz estimates, we obtain

$$
\left\|e^{i t \Delta} \phi\right\|_{S\left(\dot{H}^{1 / 2}\right)} \leq c\|\phi\|_{\dot{H}^{1 / 2}}
$$

and

$$
\left\|\int_{0}^{t} e^{i\left(t-t^{\prime}\right) \Delta} f\left(\cdot, t^{\prime}\right) d t^{\prime}\right\|_{S\left(\dot{H}^{1 / 2}\right)} \leq c\left\|D^{1 / 2} f\right\|_{S^{\prime}\left(L^{2}\right)} .
$$

\footnotetext{
${ }^{2}$ For some inequalities, the range of valid exponents $(q, r)$ can be extended. The Kato inequality (2.2) imposes the most restrictive assumptions that we incorporate into our definitions of $S\left(\dot{H}^{1 / 2}\right)$ and $S\left(\dot{H}^{-1 / 2}\right)$.
} 
We shall also need the Kato inhomogeneous Strichartz estimate [13] (for further extensions see [8] and [30])

$$
\left\|\int_{0}^{t} e^{i\left(t-t^{\prime}\right) \Delta} f\left(\cdot, t^{\prime}\right) d t^{\prime}\right\|_{S\left(\dot{H}^{1 / 2}\right)} \leq c\|f\|_{S^{\prime}\left(\dot{H}^{-1 / 2}\right)} .
$$

In particular, we will use $L_{t}^{5} L_{x}^{5}$ and $L_{t}^{20} L_{x}^{\frac{10}{3}}$ on the left side, and $L_{t}^{10 / 3} L_{x}^{5 / 4}$ on the right side.

We extend our notation $S\left(\dot{H}^{s}\right), S^{\prime}\left(\dot{H}^{s}\right)$ as follows: If a time interval is not specified (that is, if we just write $S\left(\dot{H}^{s}\right), S^{\prime}\left(\dot{H}^{s}\right)$ ), then the $t$-norm is evaluated over $(-\infty,+\infty)$. To indicate a restriction to a time subinterval $I \subset(-\infty,+\infty)$, we will write $S\left(\dot{H}^{s} ; I\right)$ or $S^{\prime}\left(\dot{H}^{s} ; I\right)$.

Proposition 2.1 (Small data). Suppose $\left\|u_{0}\right\|_{\dot{H}^{1 / 2}} \leq A$. There is $\delta_{\text {sd }}=\delta_{\text {sd }}(A)>0$ such that if $\left\|e^{i t \Delta} u_{0}\right\|_{S\left(\dot{H}^{1 / 2}\right)} \leq \delta_{\mathrm{sd}}$, then $u$ solving (1.1) is global (in $\dot{H}^{1 / 2}$ ) and

$$
\begin{gathered}
\|u\|_{S\left(\dot{H}^{1 / 2}\right)} \leq 2\left\|e^{i t \Delta} u_{0}\right\|_{S\left(\dot{H}^{1 / 2}\right)}, \\
\left\|D^{1 / 2} u\right\|_{S\left(L^{2}\right)} \leq 2 c\left\|u_{0}\right\|_{\dot{H}^{1 / 2}} .
\end{gathered}
$$

(Note that by the Strichartz estimates, the hypotheses are satisfied if $\left\|u_{0}\right\|_{\dot{H}^{1 / 2}} \leq c \delta_{\mathrm{sd}}$.)

Proof. Define

$$
\Phi_{u_{0}}(v)=e^{i t \Delta} u_{0}+i \int_{0}^{t} e^{i\left(t-t^{\prime}\right) \Delta}|v|^{2} v\left(t^{\prime}\right) d t^{\prime} .
$$

Applying the Strichartz estimates, we obtain

$$
\left\|D^{1 / 2} \Phi_{u_{0}}(v)\right\|_{S\left(L^{2}\right)} \leq c\left\|u_{0}\right\|_{\dot{H}^{1 / 2}}+c\left\|D^{1 / 2}\left(|v|^{2} v\right)\right\|_{L_{t}^{5 / 2} L_{x}^{10 / 9}}
$$

and

$$
\left\|\Phi_{u_{0}}(v)\right\|_{S\left(\dot{H}^{1 / 2}\right)} \leq\left\|e^{i t \Delta} u_{0}\right\|_{S\left(\dot{H}^{1 / 2}\right)}+c\left\|D^{1 / 2}\left(|v|^{2} v\right)\right\|_{L_{t}^{5 / 2} L_{x}^{10 / 9}} .
$$

Applying the fractional Leibnitz [17] and Hölder inequalities

$$
\left\|D^{1 / 2}\left(|v|^{2} v\right)\right\|_{L_{t}^{5 / 2} L_{x}^{10 / 9}} \leq\|v\|_{L_{t}^{5} L_{x}^{5}}^{2}\left\|D^{1 / 2} v\right\|_{L_{t}^{\infty} L_{x}^{2}} \leq\|v\|_{S\left(\dot{H}^{1 / 2}\right)}^{2}\left\|D^{1 / 2} v\right\|_{S\left(L^{2}\right)} .
$$

Let

$$
\delta_{\mathrm{sd}} \leq \min \left(\frac{1}{\sqrt{24} c}, \frac{1}{24 c A}\right)
$$

Then $\Phi_{u_{0}}: B \rightarrow B$, where

$$
B=\left\{v \mid\|v\|_{S\left(\dot{H}^{1 / 2}\right)} \leq 2\left\|e^{i t \Delta} u_{0}\right\|_{S\left(\dot{H}^{1 / 2}\right)},\left\|D^{1 / 2} v\right\|_{S\left(L^{2}\right)} \leq 2 c\left\|u_{0}\right\|_{\dot{H}^{1 / 2}}\right\}
$$

and $\Phi_{u_{0}}$ is a contraction on $B$. 
Proposition 2.2 ( $H^{1}$ scattering). If $u_{0} \in H^{1}, u(t)$ is global with globally finite $\dot{H}^{1 / 2}$ Strichartz norm $\|u\|_{S\left(\dot{H}^{1 / 2}\right)}<+\infty$ and a uniformly bounded $H^{1}$ norm $\sup _{t \in[0,+\infty)}\|u(t)\|_{H^{1}} \leq$ $B$, then $u(t)$ scatters in $H^{1}$ as $t \rightarrow+\infty$. This means that there exists $\phi^{+} \in H^{1}$ such that

$$
\lim _{t \rightarrow+\infty}\left\|u(t)-e^{i t \Delta} \phi^{+}\right\|_{H^{1}}=0 .
$$

Proof. Since $u(t)$ solves the integral equation

$$
u(t)=e^{i t \Delta} u_{0}+i \int_{0}^{t} e^{i\left(t-t^{\prime}\right) \Delta}\left(|u|^{2} u\right)\left(t^{\prime}\right) d t^{\prime},
$$

we have

$$
u(t)-e^{i t \Delta} \phi^{+}=-i \int_{t}^{+\infty} e^{i\left(t-t^{\prime}\right) \Delta}\left(|u|^{2} u\right)\left(t^{\prime}\right) d t^{\prime}
$$

where

$$
\phi^{+}=u_{0}+i \int_{0}^{+\infty} e^{-i t^{\prime} \Delta}\left(|u|^{2} u\right)\left(t^{\prime}\right) d t^{\prime} .
$$

Applying the Strichartz estimates to (2.3), we have

$$
\begin{aligned}
\left\|u(t)-e^{i t \Delta} \phi^{+}\right\|_{H^{1}} & \leq c\left\||u|^{2}(1+|\nabla|) u\right\|_{L_{[t,+\infty)}^{5 / 2} L_{x}^{10 / 9}} \\
& \leq c\|u\|_{L_{[t,+\infty}^{5} L_{x}^{5}}^{2}\|u\|_{L_{t}^{\infty} H_{x}^{1}} \\
& \leq c B\|u\|_{\left.L_{[t,+\infty}^{5}\right)_{x}^{5}}^{2} .
\end{aligned}
$$

Send $t \rightarrow+\infty$ in this inequality to obtain the claim.

The following long-time perturbation result is similar in spirit to Lemma 3.10 in Colliander-Keel-Staffilani-Takaoka-Tao [5], although more refined than a direct analogous version since the smallness condition (2.4) is expressed in terms of $S\left(\dot{H}^{1 / 2}\right)$ rather than $D^{-1 / 2} S\left(L^{2}\right)$. This refinement is achieved by employing the Kato inhomogeneous Strichartz estimates [13].

Proposition 2.3 (Long time perturbation theory). For each $A \gg 1$, there exists $\epsilon_{0}=\epsilon_{0}(A) \ll 1$ and $c=c(A) \gg 1$ such that the following holds. Let $u=u(x, t) \in H_{x}^{1}$ for all $t$ and solve

$$
i \partial_{t} u+\Delta u+|u|^{2} u=0 .
$$

Let $\tilde{u}=\tilde{u}(x, t) \in H_{x}^{1}$ for all $t$ and define

$$
e \stackrel{\text { def }}{=} i \partial_{t} \tilde{u}+\Delta \tilde{u}+|\tilde{u}|^{2} \tilde{u}
$$

If

$$
\begin{gathered}
\|\tilde{u}\|_{S\left(\dot{H}^{1 / 2}\right)} \leq A, \quad\|e\|_{S^{\prime}\left(\dot{H}^{-1 / 2}\right)} \leq \epsilon_{0}, \quad \text { and } \\
\left\|e^{i\left(t-t_{0}\right) \Delta}\left(u\left(t_{0}\right)-\tilde{u}\left(t_{0}\right)\right)\right\|_{S\left(\dot{H}^{1 / 2}\right)} \leq \epsilon_{0}
\end{gathered}
$$


then

$$
\|u\|_{S\left(\dot{H}^{1 / 2}\right)} \leq c=c(A)<\infty .
$$

Proof. Let $w$ be defined by $u=\tilde{u}+w$. Then $w$ solves the equation

$$
i \partial_{t} w+\Delta w+\left(\tilde{u}^{2} \bar{w}+2|\tilde{u}|^{2} w\right)+\left(2 \tilde{u}|w|^{2}+\overline{\tilde{u}} w^{2}\right)+|w|^{2} w-e=0 .
$$

Since $\|\tilde{u}\|_{S\left(\dot{H}^{1 / 2}\right)} \leq A$, we can partition $\left[t_{0},+\infty\right)$ into $N=N(A)$ interval ${ }^{3} I_{j}=$ $\left[t_{j}, t_{j+1}\right]$ such that for each $j$, the quantity $\|\tilde{u}\|_{S\left(\dot{H}^{1 / 2} ; I_{j}\right)} \leq \delta$ is suitably small ( $\delta$ to be chosen below). The integral equation version of (2.5) with initial time $t_{j}$ is

$$
w(t)=e^{i\left(t-t_{j}\right) \Delta} w\left(t_{j}\right)+i \int_{t_{j}}^{t} e^{i(t-s) \Delta} W(\cdot, s) d s
$$

where

$$
W=\left(\tilde{u}^{2} \bar{w}+2|\tilde{u}|^{2} w\right)+\left(2 \tilde{u}|w|^{2}+\overline{\tilde{u}} w^{2}\right)+|w|^{2} w-e .
$$

By applying the Kato Strichartz estimate (2.2) on $I_{j}$, we obtain

$$
\begin{aligned}
\|w\|_{S\left(\dot{H}^{1 / 2} ; I_{j}\right)} & \leq\left\|e^{i\left(t-t_{j}\right) \Delta} w\left(t_{j}\right)\right\|_{S\left(\dot{H}^{1 / 2} ; I_{j}\right)}+c\left\|\tilde{u}^{2} w\right\|_{L_{I_{j}}^{10 / 3} L_{x}^{5 / 4}} \\
& +c\left\|\tilde{u} w^{2}\right\|_{L_{I_{j}}^{10 / 3} L_{x}^{5 / 4}}+c\left\|w^{3}\right\|_{L_{I_{j}}^{10 / 3} L_{x}^{5 / 4}}+\|e\|_{S^{\prime}\left(\dot{H}^{-1 / 2} ; I_{j}\right)} .
\end{aligned}
$$

Observe

$$
\left\|\tilde{u}^{2} w\right\|_{L_{I_{j}}^{10 / 3} L_{x}^{5 / 4}} \leq\|\tilde{u}\|_{L_{I_{j}}^{20} L_{x}^{10 / 3}}^{2}\|w\|_{L_{I_{j}}^{5} L_{x}^{5}} \leq\|\tilde{u}\|_{S\left(\dot{H}^{1 / 2} ; I_{j}\right)}^{2}\|w\|_{S\left(\dot{H}^{1 / 2} ; I_{j}\right)} \leq \delta^{2}\|w\|_{S\left(\dot{H}^{1 / 2} ; I_{j}\right)}
$$

Similarly,

$$
\left\|\tilde{u} w^{2}\right\|_{L_{I_{j}}^{10 / 3} L_{x}^{5 / 4}} \leq \delta\|w\|_{S\left(\dot{H}^{1 / 2} ; I_{j}\right)}^{2}, \quad \text { and } \quad\left\|w^{3}\right\|_{L_{I_{j}}^{10 / 3} L_{x}^{5 / 4}} \leq\|w\|_{S\left(\dot{H}^{1 / 2} ; I_{j}\right)}^{3} .
$$

Substituting the above estimates in (2.7),

$$
\begin{aligned}
\|w\|_{S\left(\dot{H}^{1 / 2} ; I_{j}\right)} & \leq\left\|e^{i\left(t-t_{j}\right) \Delta} w\left(t_{j}\right)\right\|_{S\left(\dot{H}^{1 / 2} ; I_{j}\right)}+c \delta^{2}\|w\|_{S\left(\dot{H}^{1 / 2} ; I_{j}\right)} \\
& +c \delta\|w\|_{S\left(\dot{H}^{1 / 2} ; I_{j}\right)}^{2}+c\|w\|_{S\left(\dot{H}^{1 / 2} ; I_{j}\right)}^{3}+c \epsilon_{0} .
\end{aligned}
$$

Provided

$$
\delta \leq \min \left(1, \frac{1}{6 c}\right) \quad \text { and } \quad\left(\left\|e^{i\left(t-t_{j}\right) \Delta} w\left(t_{j}\right)\right\|_{S\left(\dot{H}^{1 / 2} ; I_{j}\right)}+c \epsilon_{0}\right) \leq \min \left(1, \frac{1}{2 \sqrt{6 c}}\right)
$$

we obtain

$$
\|w\|_{S\left(\dot{H}^{1 / 2} ; I_{j}\right)} \leq 2\left\|e^{i\left(t-t_{j}\right) \Delta} w\left(t_{j}\right)\right\|_{S\left(\dot{H}^{1 / 2} ; I_{j}\right)}+2 c \epsilon_{0} .
$$

Now take $t=t_{j+1}$ in (2.6) , and apply $e^{i\left(t-t_{j+1}\right) \Delta}$ to both sides to obtain

$$
e^{i\left(t-t_{j+1}\right) \Delta} w\left(t_{j+1}\right)=e^{i\left(t-t_{j}\right) \Delta} w\left(t_{j}\right)+i \int_{t_{j}}^{t_{j+1}} e^{i(t-s) \Delta} W(\cdot, s) d s .
$$

\footnotetext{
${ }^{3}$ The number of intervals depends only on $A$, but the intervals themselves depend upon the function $\tilde{u}$.
} 
Since the Duhamel integral is confined to $I_{j}=\left[t_{j}, t_{j+1}\right]$, by again applying the Kato estimate, similarly to (2.8) we obtain the estimate

$$
\begin{aligned}
\left\|e^{i\left(t-t_{j+1}\right) \Delta} w\left(t_{j+1}\right)\right\|_{S\left(\dot{H}^{1 / 2}\right)} & \leq\left\|e^{i\left(t-t_{j}\right) \Delta} w\left(t_{j}\right)\right\|_{S\left(\dot{H}^{1 / 2}\right)}+c \delta^{2}\|w\|_{S\left(\dot{H}^{1 / 2} ; I_{j}\right)} \\
& +c \delta\|w\|_{S\left(\dot{H}^{1 / 2} ; I_{j}\right)}^{2}+c\|w\|_{S\left(\dot{H}^{1 / 2} ; I_{j}\right)}^{3}+c \epsilon_{0} .
\end{aligned}
$$

By (2.10) and (2.11), we bound the previous expression to obtain

$$
\left\|e^{i\left(t-t_{j+1}\right) \Delta} w\left(t_{j+1}\right)\right\|_{S\left(\dot{H}^{1 / 2}\right)} \leq 2\left\|e^{i\left(t-t_{j}\right) \Delta} w\left(t_{j}\right)\right\|_{S\left(\dot{H}^{1 / 2}\right)}+2 c \epsilon_{0} .
$$

Iterating beginning with $j=0$, we obtain

$$
\begin{aligned}
\left\|e^{i\left(t-t_{j}\right) \Delta} w\left(t_{j}\right)\right\|_{S\left(\dot{H}^{1 / 2}\right)} & \leq 2^{j}\left\|e^{i\left(t-t_{0}\right) \Delta} w\left(t_{0}\right)\right\|_{S\left(\dot{H}^{1 / 2}\right)}+\left(2^{j}-1\right) 2 c \epsilon_{0} \\
& \leq 2^{j+2} c \epsilon_{0} .
\end{aligned}
$$

To accommodate the second part of (2.9) for all intervals $I_{j}, 0 \leq j \leq N-1$, we require that

$$
2^{N+2} c \epsilon_{0} \leq \min \left(1, \frac{1}{2 \sqrt{6 c}}\right)
$$

We review the dependence of parameters: $\delta$ is an absolute constant selected to meet the first part of (2.9). We were given $A$, which then determined $N$ (the number of time subintervals). The inequality (2.12) specifies how small $\epsilon_{0}$ needs to be taken in terms of $N$ (and thus, in terms of $A$ ).

\section{Properties of the ground state}

M. Weinstein [31] proved that the sharp constant $c_{\mathrm{GN}}$ in the Gagliardo-Nirenberg estimate

$$
\|f\|_{L^{4}}^{4} \leq c_{\mathrm{GN}}\|f\|_{L^{2}}\|\nabla f\|_{L^{2}}^{3}
$$

is attained at the function $Q$ (the ground state described in the introduction), i.e., $c_{\mathrm{GN}}=\|Q\|_{L^{4}}^{4} /\left(\|Q\|_{L^{2}}\|\nabla Q\|_{L^{2}}^{3}\right)$. By multiplying (1.2) by $Q$, integrating, and applying integration by parts, we obtain

$$
-\|Q\|_{L^{2}}^{2}-\|\nabla Q\|_{L^{2}}^{2}+\|Q\|_{L^{4}}^{4}=0
$$

By multiplying (1.2) by $x \cdot \nabla Q$, integrating, and applying integration by parts, we obtain the Pohozhaev identity

$$
\frac{3}{2}\|Q\|_{L^{2}}^{2}+\frac{1}{2}\|\nabla Q\|_{L^{2}}^{2}-\frac{3}{4}\|Q\|_{L^{4}}^{4}=0 .
$$

These two identities enable us to obtain the relations

$$
\|\nabla Q\|_{L^{2}}^{2}=3\|Q\|_{L^{2}}^{2}, \quad\|Q\|_{L^{4}}^{4}=4\|Q\|_{L^{2}}^{2},
$$


and thus, reexpress

$$
c_{\mathrm{GN}}=\frac{4}{3\|Q\|_{L^{2}}\|\nabla Q\|_{L^{2}}}=\frac{4}{3 \sqrt{3}\|Q\|_{L^{2}}^{2}}
$$

We also calculate

$$
M[Q] E[Q]=\|Q\|_{L^{2}}^{2}\left(\frac{1}{2}\|\nabla Q\|_{L^{2}}^{2}-\frac{1}{4}\|Q\|_{L^{4}}^{4}\right)=\frac{1}{6}\|Q\|_{L^{2}}^{2}\|\nabla Q\|_{L^{2}}^{2}=\frac{1}{2}\|Q\|_{L^{2}}^{4} .
$$

For later purposes we recall a version of the Gagliardo-Nirenberg inequality valid only for radial functions, due to W. Strauss [25]. In $\mathbb{R}^{3}$, for any $R>0$, we have

$$
\|f\|_{L^{4}(|x|>R)}^{4} \leq \frac{c}{R^{2}}\|f\|_{L^{2}(|x|>R)}^{3}\|\nabla f\|_{L^{2}(|x|>R)} .
$$

\section{Global Versus Blow-up Dichotomy}

In this section we show how to obtain Theorem 1.1part (11) (a) and part(2). This was proved in Holmer-Roudenko [12 for general mass supercritical and energy subcritical NLS equations with $H^{1}$ initial data, but for self-containment of this exposition we outline the main ideas here.

Before giving the proof, we observe that the following quantities are scaling invariant:

$$
\|\nabla u\|_{L^{2}} \cdot\|u\|_{L^{2}} \quad \text { and } \quad E[u] \cdot M[u] .
$$

Next, we quote a localized version of the virial identity as in Kenig-Merle [16]. We refer, for example, to Merle-Raphaël [21] or Ozawa-Tsutsumi [22] for a proof.

Lemma 4.1 (Local virial identity). Let $\chi \in C_{0}^{\infty}\left(\mathbb{R}^{N}\right)$, radially symmetric and u solve

$$
i \partial_{t} u+\Delta u+|u|^{p-1} u=0 \text {. }
$$

Then

$$
\partial_{t}^{2} \int \chi(x)|u(x, t)|^{2} d x=4 \int \chi^{\prime \prime}|\nabla u|^{2}-\int \Delta^{2} \chi|u|^{2}-4\left(\frac{1}{2}-\frac{1}{p+1}\right) \int \Delta \chi|u|^{p+1}
$$

We prove a slightly stronger version of Theorem 1.1 parts (11)(a) and (2) that is valid for nonradial initial condition. The generalization of this theorem to all mass supercritical and energy critical cases of NLS can be found in $\$ 7$ as well as in [12. A different type of condition for global existence, phrased as $\left\|u_{0}\right\|_{L^{2}} \leq \gamma_{*}\left(\left\|\nabla u_{0}\right\|_{L^{2}}\right)$ for a certain monotonic function $\gamma: \mathbb{R}_{+} \rightarrow \mathbb{R}_{+}$, is given by Bégout [2].

Theorem 4.2 (Global versus blow-up dichotomy). Let $u_{0} \in H^{1}\left(\mathbb{R}^{3}\right)$ (possibly nonradial), and let $I=\left(-T_{*}, T^{*}\right)$ be the maximal time interval of existence of $u(t)$ solving (1.1). Suppose that

$$
M\left[u_{0}\right] E\left[u_{0}\right]<M[Q] E[Q] .
$$

\footnotetext{
${ }^{4}$ Numerical calculations show $\|Q\|_{L^{2}\left(\mathbb{R}^{3}\right)}^{2} \cong 18.94$, which gives $c_{\mathrm{GN}} \cong 0.0406$ (in $\left.\mathbb{R}^{3}\right)$.
} 
If (4.2) holds and

$$
\left\|\nabla u_{0}\right\|_{L^{2}}\left\|u_{0}\right\|_{L^{2}}<\|\nabla Q\|_{L^{2}}\|Q\|_{L^{2}}
$$

then $I=(-\infty,+\infty)$, i.e. the solution exists globally in time, and for all time $t \in \mathbb{R}$

$$
\|\nabla u(t)\|_{L^{2}}\left\|u_{0}\right\|_{L^{2}}<\|\nabla Q\|_{L^{2}}\|Q\|_{L^{2}}
$$

If (4.2) holds and

$$
\left\|\nabla u_{0}\right\|_{L^{2}}\left\|u_{0}\right\|_{L^{2}}>\|\nabla Q\|_{L^{2}}\|Q\|_{L^{2}}
$$

then for $t \in I$

$$
\|\nabla u(t)\|_{L^{2}}\left\|u_{0}\right\|_{L^{2}}>\|\nabla Q\|_{L^{2}}\|Q\|_{L^{2}} .
$$

Furthermore, if (a) $|x| u_{0} \in L^{2}\left(\mathbb{R}^{3}\right)$, or (b) $u_{0}$ is radial, then $I$ is finite, and thus, the solution blows up in finite time.

We recently became aware that the global existence assertion and the blow-up assertion under the hypothesis $|x| u_{0} \in L^{2}\left(\mathbb{R}^{3}\right)$ in this theorem previously appeared in the literature in Kuznetsov-Rasmussen-Rypdal-Turitsyn [19]. We have decided to keep the proof below since it is short and for the convenience of the reader (there are significant notational differences between our paper and theirs).

Remark 4.3. 5 Since this theorem applies to the nonradial case, we remark that one should exploit the Galilean invariance to extend the class of solutions $u$ to which it applies. Since $u$ is global [respectively, blows up in finite time] if and only if a Galilean transformation of it is global [respectively, blows up in finite time], given $u$ consider for some $\xi_{0} \in \mathbb{R}^{3}$ the transformed solution

$$
w(x, t)=e^{i x \cdot \xi_{0}} e^{-i t\left|\xi_{0}\right|^{2}} u\left(x-2 \xi_{0} t, t\right) .
$$

We compute

$$
\|\nabla w\|_{L^{2}}^{2}=\left|\xi_{0}\right|^{2} M[u]+2 \xi_{0} \cdot P[u]+\|\nabla u\|_{L^{2}}^{2},
$$

where the vector $P[u]=\operatorname{Im} \int \bar{u} \nabla u d x$ is the conserved momentum. Therefore, $M[w]=$ $M[u]$ and

$$
E[w]=\frac{1}{2}\left|\xi_{0}\right|^{2} M[u]+\xi_{0} \cdot P[u]+E[u] .
$$

To minimize $E[w]$ and $\|\nabla w\|_{L^{2}}$, we take $\xi_{0}=-P[u] / M[u]$. Then we test the condition (4.2), and (4.3) or (4.5) for $w$, rather than $u$. This means that for $P \neq 0$, the hypothesis (4.2) can be sharpened to

$$
M[u]\left(-\frac{P[u]^{2}}{2 M[u]}+E[u]\right)<M[Q] E[Q]
$$

\footnotetext{
${ }^{5}$ We thank J. Colliander for supplying this comment.
} 
and the hypothesis (4.3) can be sharpened to

$$
\left(-\frac{P[u]^{2}}{M[u]}+\left\|\nabla u_{0}\right\|_{L^{2}}^{2}\right)\left\|u_{0}\right\|_{L^{2}}^{2}<\|Q\|_{L^{2}}^{2}\|\nabla Q\|_{L^{2}}^{2}
$$

and similarly for (4.5).

Proof. Multiplying the definition of energy by $M[u]$ and using (3.1), we have

$$
\begin{aligned}
M[u] E[u] & =\frac{1}{2}\|\nabla u\|_{L^{2}}^{2}\left\|u_{0}\right\|_{L^{2}}^{2}-\frac{1}{4}\|u\|_{L^{4}}^{4}\left\|u_{0}\right\|_{L^{2}}^{2} \\
& \geq \frac{1}{2}\|\nabla u\|_{L^{2}}^{2}\left\|u_{0}\right\|_{L^{2}}^{2}-\frac{1}{4} c_{\mathrm{GN}}\|\nabla u\|_{L^{2}}^{3}\left\|u_{0}\right\|_{L^{2}}^{3} .
\end{aligned}
$$

Define $f(x)=\frac{1}{2} x^{2}-\frac{c_{\mathrm{GN}}}{4} x^{3}$. Then $f^{\prime}(x)=x-\frac{3}{4} c_{\mathrm{GN}} x^{2}=x\left(1-\frac{3}{4} c_{\mathrm{GN}} x\right)$, and thus, $f^{\prime}(x)=0$ when $x_{0}=0$ and $x_{1}=\frac{4}{3} \frac{1}{c_{\mathrm{GN}}}=\|\nabla Q\|_{L^{2}}\|Q\|_{L^{2}}$ by (3.3). Note that $f(0)=0$ and $f\left(x_{1}\right)=\frac{1}{6}\|\nabla Q\|_{L^{2}}^{2}\|Q\|_{L^{2}}^{2}$. Thus, the graph of $f$ has a local minimum at $x_{0}$ and a local maximum at $x_{1}$. The condition (4.2) together with (3.4) imply that $M\left[u_{0}\right] E\left[u_{0}\right]<f\left(x_{1}\right)$. Combining this with energy conservation, we have

$$
f\left(\|\nabla u(t)\|_{L^{2}}\left\|u_{0}\right\|_{L^{2}}\right) \leq M\left[u_{0}\right] E[u(t)]=M\left[u_{0}\right] E\left[u_{0}\right]<f\left(x_{1}\right) .
$$

If initially $\left\|u_{0}\right\|_{L^{2}}\left\|\nabla u_{0}\right\|_{L^{2}}<x_{1}$, i.e. the condition (4.3) holds, then by (4.7) and the continuity of $\|\nabla u(t)\|_{L^{2}}$ in $t$, we have $\left\|u_{0}\right\|_{L^{2}}\|\nabla u(t)\|_{L^{2}}<x_{1}$ for all time $t \in I$ which gives (4.4). In particular, the $\dot{H}^{1}$ norm of the solution $u$ is bounded, which proves global existence (and thus, global wellposedness) in this case.

If initially $\left\|u_{0}\right\|_{L^{2}}\left\|\nabla u_{0}\right\|_{L^{2}}>x_{1}$, i.e. the condition (4.5) holds, then by (4.7) and the continuity of $\|\nabla u(t)\|_{L^{2}}$ in $t$, we have $\left\|u_{0}\right\|_{L^{2}}\|\nabla u(t)\|_{L^{2}}>x_{1}$ for all time $t \in I$ which gives (4.6). We can refine this analysis to obtain the following: if the condition (4.5) (together with (4.2)) holds, then there exists $\delta_{1}>0$ such that $M\left[u_{0}\right] E\left[u_{0}\right]<\left(1-\delta_{1}\right) M[Q] E[Q]$, and thus, there exists $\delta_{2}=\delta_{2}\left(\delta_{1}\right)>0$ such that $\left\|u_{0}\right\|_{L^{2}}^{2}\|\nabla u(t)\|_{L^{2}}^{2}>\left(1+\delta_{2}\right)\|\nabla Q\|_{L^{2}}^{2}\|Q\|_{L^{2}}^{2}$ for all $t \in I$.

Now if $u$ has a finite variance, we recall the virial identity

$$
\partial_{t}^{2} \int|x|^{2}|u(x, t)|^{2} d x=24 E\left[u_{0}\right]-4\|\nabla u(t)\|_{L^{2}}^{2}
$$

Multiplying both sides by $M\left[u_{0}\right]$ and applying the refinement of inequalities (4.2) and (4.6) mentioned above as well as (3.4), we get

$$
\begin{aligned}
M\left[u_{0}\right] \partial_{t}^{2} \int|x|^{2}|u(x, t)|^{2} d x & =24 M\left[u_{0}\right] E\left[u_{0}\right]-4\|\nabla u(t)\|_{L^{2}}^{2}\left\|u_{0}\right\|_{L^{2}}^{2} \\
& <24 \cdot \frac{1}{6}\left(1-\delta_{1}\right)\|\nabla Q\|_{L^{2}}^{2}\|Q\|_{L^{2}}^{2}-4\left(1+\delta_{2}\right)\|\nabla Q\|_{L^{2}}^{2}\|Q\|_{L^{2}}^{2} \\
& =-4\left(\delta_{1}+\delta_{2}\right)\|\nabla Q\|_{L^{2}}^{2}\|Q\|_{L^{2}}^{2}<0,
\end{aligned}
$$

and thus, $I$ must be finite, which implies that blow up occurs in finite time.

If $u_{0}$ is radial, we use a localized version of the virial identity (4.1). Choose $\chi(r)$ (radial) such that $\partial_{r}^{2} \chi(r) \leq 2$ for all $r \geq 0, \chi(r)=r^{2}$ for $0 \leq r \leq 1$, and $\chi(r)$ is 
constant for $r \geq 3$. Let $\chi_{m}(r)=m^{2} \chi(r / m)$. The rest of the argument follows the proof of the main theorem in Ogawa-Tsutsumi [22], although we include the details here for the convenience of the reader. We bound each of the terms in the local virial identity (4.1) as follows, using that $\Delta \chi_{m}(r)=6$ for $r \leq m$ and $\Delta^{2} \chi_{m}(r)=0$ for $r \leq m$ :

$$
\begin{aligned}
& 4 \int \chi_{m}^{\prime \prime}|\nabla u|^{2} \\
-\int \Delta^{2} \chi_{m}|u|^{2} & \leq \frac{c}{m^{2}} \int_{m \leq|x| \leq 3 m}|\nabla u|^{2}, \\
- & |u|^{2}, \\
-\int \Delta \chi_{m}|u|^{4} & \leq-6 \int_{|x| \leq m}|u|^{4}+c \int_{m \leq|x| \leq 3 m}|u|^{4} \leq-6 \int_{\mathbb{R}^{3}}|u|^{4}+c^{\prime} \int_{|x| \geq m}|u|^{4} .
\end{aligned}
$$

Adding these three bounds and applying the radial Gagliardo-Nirenberg estimate (3.5), we obtain that for any large $m>0$, we have

$$
\begin{aligned}
\partial_{t}^{2} \int \chi_{m}(|x|)|u(x, t)|^{2} d x \leq & 24 E\left[u_{0}\right]-4 \int|\nabla u|^{2} \\
& +\frac{c_{1}}{m^{2}}\left\|u_{0}\right\|_{L^{2}}^{3}\|\nabla u\|_{L^{2}}+\frac{c_{2}}{m^{2}} \int_{m<|x|}|u|^{2} .
\end{aligned}
$$

Let $\epsilon>0$ be a small constant to be chosen below. Use Young's inequality in the third term on the right side to separate the $L^{2}$-norm and gradient term and then absorb the gradient term into the second term with the chosen $\epsilon$. Multiplying the above expression by $M\left[u_{0}\right]$, we get

$$
\begin{aligned}
& M\left[u_{0}\right] \partial_{t}^{2} \int \chi_{m}(|x|)|u(x, t)|^{2} d x \\
& \quad \leq 24 E\left[u_{0}\right] M\left[u_{0}\right]-(4-\epsilon)\|\nabla u\|_{L^{2}}^{2}\left\|u_{0}\right\|_{L^{2}}^{2}+\frac{c(\epsilon)}{m^{4}}\left\|u_{0}\right\|_{L^{2}}^{8}+\frac{c_{2}}{m^{2}}\left\|u_{0}\right\|_{L^{2}}^{4} \\
& \quad \leq-c_{3}\|\nabla Q\|_{L^{2}}\|Q\|_{L^{2}}+\frac{c(\epsilon)}{m^{4}}\left\|u_{0}\right\|_{L^{2}}^{8}+\frac{c_{2}}{m^{2}}\left\|u_{0}\right\|_{L^{2}}^{4},
\end{aligned}
$$

where

$$
c_{3} \equiv-4\left(1-\delta_{1}\right)+(4-\epsilon)\left(1+\delta_{2}\right)=+4 \delta_{1}-\epsilon\left(1+\delta_{2}\right) .
$$

Select $\epsilon=\epsilon\left(\delta_{1}, \delta_{2}\right)>0$ so that $c_{3}>0$ and then take $m=m\left(c_{3}, \epsilon, M\left[u_{0}\right]\right)$ large enough so that the right side of (4.8) is bounded by a strictly negative constant. This implies that the maximal interval of existence $I$ is finite.

The next two lemmas provide some additional estimates that hold under the hypotheses (4.2) and (4.3) of Theorem 4.2. These estimates will be needed for the compactness and rigidity results in $\$ 5,6$.

Lemma 4.4 (Lower bound on the convexity of the variance). Let $u_{0} \in H^{1}\left(\mathbb{R}^{3}\right)$ satisfy (4.2) and (4.3). Furthermore, take $\delta>0$ such that $M\left[u_{0}\right] E\left[u_{0}\right]<(1-\delta) M[Q] E[Q]$. 
If $u$ is the solution of the Cauchy problem (1.1) with initial data $u_{0}$, then there exists $c_{\delta}>0$ such that for all $t \in \mathbb{R}$

$$
24 E[u]-4\|\nabla u(t)\|_{L^{2}}^{2}=8\|\nabla u(t)\|_{L^{2}}^{2}-6\|u(t)\|_{L^{4}}^{4} \geq c_{\delta}\|\nabla u(t)\|_{L^{2}}^{2} .
$$

Proof. By the analysis in the proof of Theorem 4.2, there exists $\delta_{2}=\delta_{2}(\delta)>0$ such that for all $t \in \mathbb{R}$,

$$
\left\|u_{0}\right\|_{L^{2}}^{2}\|\nabla u(t)\|_{L^{2}}^{2} \leq\left(1-\delta_{2}\right)^{2}\|\nabla Q\|_{L^{2}}^{2}\|Q\|_{L^{2}}^{2}
$$

Let

$$
h(t)=\frac{1}{\|Q\|_{L^{2}}^{2}\|\nabla Q\|_{L^{2}}^{2}}\left(8\left\|u_{0}\right\|_{L^{2}}^{2}\|\nabla u(t)\|_{L^{2}}^{2}-6\left\|u_{0}\right\|_{L^{2}}^{2}\|u(t)\|_{L^{4}}^{4}\right),
$$

and set $g(y)=y^{2}-y^{3}$. By the Gagliardo-Nirenberg estimate (3.1) with sharp constant $c_{\mathrm{GN}}$ and (3.3),

$$
h(t) \geq 8 g\left(\frac{\|\nabla u(t)\|_{L^{2}}\left\|u_{0}\right\|_{L^{2}}}{\|\nabla Q\|_{L^{2}}\|Q\|_{L^{2}}}\right) .
$$

By (4.10), we restrict attention to $0 \leq y \leq 1-\delta_{2}$. By an elementary argument, there exists $c=c\left(\delta_{2}\right)$ such that $g(y) \geq c y^{2}$ if $0 \leq y \leq 1-\delta_{2}$, which completes the proof.

Lemma 4.5 (Comparability of gradient and energy). Let $u_{0} \in H^{1}\left(\mathbb{R}^{3}\right)$ satisfy (4.2) and (4.3). Then

$$
\frac{1}{6}\|\nabla u(t)\|_{L^{2}}^{2} \leq E[u] \leq \frac{1}{2}\|\nabla u(t)\|_{L^{2}}^{2} .
$$

Proof. The second inequality is immediate from the definition of energy. The first one is obtained by observing that

$$
\frac{1}{2}\|\nabla u\|_{L^{2}}^{2}-\frac{1}{4}\|u\|_{L^{4}}^{4} \geq \frac{1}{2}\|\nabla u\|_{L^{2}}^{2}\left(1-\frac{1}{2} c_{\mathrm{GN}}\|\nabla u\|_{L^{2}}\|u\|_{L^{2}}\right) \geq \frac{1}{6}\|\nabla u\|_{L^{2}}^{2},
$$

where we used (3.1), (3.3) and (4.4).

In the proofs of Proposition 5.4 and 5.5, we will need the following result called existence of wave operators since the map $\Omega^{+}: \psi^{+} \mapsto v_{0}$ is called the wave operator (see the proposition for the meaning of $\psi^{+}$and $v_{0}$ ).

Proposition 4.6 (Existence of wave operators). Suppose $\psi^{+} \in H^{1}$ and

$$
\frac{1}{2}\left\|\psi^{+}\right\|_{L^{2}}^{2}\left\|\nabla \psi^{+}\right\|_{L^{2}}^{2}<M[Q] E[Q] .
$$

Then there exists $v_{0} \in H^{1}$ such that $v$ solving (1.1) with initial data $v_{0}$ is global in $H^{1}$ with

$$
\|\nabla v(t)\|_{L^{2}}\left\|v_{0}\right\|_{L^{2}} \leq\|Q\|_{L^{2}}\|\nabla Q\|_{L^{2}}, \quad M[v]=\left\|\psi^{+}\right\|_{L^{2}}^{2}, \quad E[v]=\frac{1}{2}\left\|\nabla \psi^{+}\right\|_{L^{2}}^{2}
$$

and

$$
\lim _{t \rightarrow+\infty}\left\|v(t)-e^{i t \Delta} \psi^{+}\right\|_{H^{1}}=0
$$


Moreover, if $\left\|e^{i t \Delta} \psi^{+}\right\|_{S\left(\dot{H}^{1 / 2}\right)} \leq \delta_{\mathrm{sd}}$, then

$$
\left\|v_{0}\right\|_{\dot{H}^{1 / 2}} \leq 2\left\|\psi^{+}\right\|_{\dot{H}^{1 / 2}} \quad \text { and } \quad\|v\|_{S\left(\dot{H}^{1 / 2}\right)} \leq 2\left\|e^{i t \Delta} \psi^{+}\right\|_{S\left(\dot{H}^{1 / 2}\right)} .
$$

Proof. We want to solve the integral equation

$$
v(t)=e^{i t \Delta} \psi^{+}-i \int_{t}^{+\infty} e^{i\left(t-t^{\prime}\right) \Delta}\left(|v|^{2} v\right)\left(t^{\prime}\right) d t^{\prime}
$$

first for $t \geq T$ with $T$ large. This is achieved as in the proof of the small data scattering theory (Proposition 2.1), since there exists $T \gg 0$ such that $\left\|e^{i t \Delta} \psi^{+}\right\|_{S\left(\dot{H}^{1 / 2} ;[T,+\infty)\right)} \leq$ $\delta_{\text {sd }}$. By estimating (4.12), we obtain

$$
\begin{aligned}
\|\nabla v\|_{S\left(L^{2} ;[T,+\infty)\right)} & \leq c\left\|\psi^{+}\right\|_{\dot{H}^{1}}+c\left\|\nabla\left(v^{3}\right)\right\|_{S^{\prime}\left(L^{2}\right)} \\
& \leq c\left\|\psi^{+}\right\|_{\dot{H}^{1}}+c\|\nabla v\|_{S\left(L^{2} ;[T,+\infty)\right)}\|v\|_{\left.S\left(\dot{H}^{1 / 2} ; T,+\infty\right)\right)}^{2}
\end{aligned}
$$

where in the last step, we used $\|\cdot\|_{S^{\prime}\left(L^{2}\right)} \leq\|\cdot\|_{L_{t}^{10 / 7} L_{x}^{10 / 7}}$ and the Hölder partition $\frac{7}{10}=\frac{3}{10}+\frac{1}{5}+\frac{1}{5}$. Thus, $\|\nabla v\|_{S\left(L^{2} ;[T,+\infty)\right)} \leq 2 c\left\|\psi^{+}\right\|_{\dot{H}^{1}}$. Using this, we obtain similarly,

$$
\left\|\nabla\left(v(t)-e^{i t \Delta} \psi^{+}\right)\right\|_{S\left(L^{2} ;[T,+\infty)\right)} \rightarrow 0 \text { as } T \rightarrow+\infty .
$$

Since $v(t)-e^{i t \Delta} \psi^{+} \rightarrow 0$ in $H^{1}$ as $t \rightarrow+\infty, e^{i t \Delta} \psi^{+} \rightarrow 0$ in $L^{4}$ as $t \rightarrow+\infty$, and $\left\|\nabla e^{i t \Delta} \psi^{+}\right\|_{L^{2}}$ is conserved, we have

$$
E[v]=\lim _{t \rightarrow+\infty}\left(\frac{1}{2}\left\|\nabla e^{i t \Delta} \psi^{+}\right\|_{L^{2}}^{2}-\frac{1}{4}\left\|e^{i t \Delta} \psi^{+}\right\|_{L^{4}}^{4}\right)=\frac{1}{2}\left\|\psi^{+}\right\|_{L^{2}}^{2} .
$$

Immediately, we obtain $M[v]=\left\|\psi^{+}\right\|_{L^{2}}^{2}$. Note that we now have $M[v] E[v]<$ $M[Q] E[Q]$ by (4.11). Observe that

$$
\begin{aligned}
\lim _{t \rightarrow+\infty}\|\nabla v(t)\|_{L^{2}}^{2}\|v\|_{L^{2}}^{2} & =\lim _{t \rightarrow+\infty}\left\|\nabla e^{i t \Delta} \psi^{+}\right\|_{L^{2}}^{2}\left\|e^{i t \Delta} \psi^{+}\right\|_{L^{2}}^{2} \\
& =\left\|\nabla \psi^{+}\right\|_{L^{2}}^{2}\left\|\psi^{+}\right\|_{L^{2}}^{2} \\
& \leq 2 M[Q] E[Q] \\
& =\frac{1}{3}\|\nabla Q\|_{L^{2}}^{2}\|Q\|_{L^{2}}^{2},
\end{aligned}
$$

where in the last two steps we used (4.11) and (3.4). Take $T$ sufficiently large so that $\|\nabla v(T)\|_{L^{2}}\|v\|_{L^{2}} \leq\|\nabla Q\|_{L^{2}}\|Q\|_{L^{2}}$. By Theorem 4.2, we can evolve $v(t)$ from $T$ back to time 0 .

\section{Compactness}

Definition 5.1. Suppose $u_{0} \in H^{1}$ and $u$ is the corresponding $H^{1}$ solution to (1.1) and $\left[0, T^{*}\right)$ the maximal forward time interval of existence. We shall say that $\mathrm{SC}\left(u_{0}\right)$ holds if $T^{*}=+\infty$ and $\|u\|_{S\left(\dot{H}^{1 / 2}\right)}<\infty$. 
To prove Theorem1.1(1) (b), we must show that if $\left\|u_{0}\right\|_{L^{2}}\left\|\nabla u_{0}\right\|_{L^{2}}<\|Q\|_{L^{2}}\|\nabla Q\|_{L^{2}}$, and $M[u] E[u]<M[Q] E[Q]$, then $\mathrm{SC}\left(u_{0}\right)$ holds. We already know that under these hypotheses, we have an a priori bound on $\|\nabla u(t)\|_{L^{2}}$, and thus, the maximal forward time of existence is $T^{*}=+\infty$ (this is the energy subcritical case). The goal is, therefore, to show that the global-in-time $\dot{H}^{1 / 2}$ Strichartz norm is finite.

By Lemma 4.5, if $M[u] E[u]<\frac{1}{6} \delta_{\text {sd }}^{4}$ and $\left\|u_{0}\right\|_{L^{2}}\left\|\nabla u_{0}\right\|_{L^{2}}<\|Q\|_{L^{2}}\|\nabla Q\|_{L^{2}}$, then

$$
\left\|u_{0}\right\|_{\dot{H}^{1 / 2}}^{4} \leq\left\|u_{0}\right\|_{L^{2}}^{2}\left\|\nabla u_{0}\right\|_{L^{2}}^{2} \leq 6 M[u] E[u] \leq \delta_{\text {sd }}^{4} .
$$

Thus, by the small data theory (Proposition 2.1), $\mathrm{SC}\left(u_{0}\right)$ holds. Let $(M E)_{\mathrm{c}}$ be the number defined as the supremum over all $\delta$ for which the following statement holds true: "If $u_{0}$ is radial with $\left\|u_{0}\right\|_{L^{2}}\left\|\nabla u_{0}\right\|_{L^{2}}<\|Q\|_{L^{2}}\|\nabla Q\|_{L^{2}}$ and $M[u] E[u]<\delta$, then $\mathrm{SC}\left(u_{0}\right)$ holds." We then clearly have $0<\frac{1}{6} \delta_{\mathrm{sd}}^{4} \leq(M E)_{\mathrm{c}}$. If $(M E)_{\mathrm{c}} \geq M[Q] E[Q]$, then Theorem 1.1(1) (b) is true. We, therefore, proceed with the proof of Theorem 1.1(1) (b) by assuming that $(M E)_{\mathrm{c}}<M[Q] E[Q]$ and ultimately deduce a contradiction (much later, in $\$ 6$ ). By definition of $(M E)_{\mathrm{c}}$, we have

(C.1) If $u_{0}$ is radial and $\left\|u_{0}\right\|_{L^{2}}\left\|\nabla u_{0}\right\|_{L^{2}}<\|Q\|_{L^{2}}\|\nabla Q\|_{L^{2}}$ and $M[u] E[u]<(M E)_{\mathrm{c}}$, then $\mathrm{SC}\left(u_{0}\right)$ holds.

(C.2) There exists a sequence of radial solutions $u_{n}$ to (1.1) with corresponding initial data $u_{n, 0}$ such that $\left\|u_{n, 0}\right\|_{L^{2}}\left\|\nabla u_{n, 0}\right\|_{L^{2}}<\|Q\|_{L^{2}}\|\nabla Q\|_{L^{2}}$ and $M\left[u_{n}\right] E\left[u_{n}\right] \searrow$ $(M E)_{\mathrm{c}}$ as $n \rightarrow+\infty$, for which $\mathrm{SC}\left(u_{n, 0}\right)$ does not hold for any $n$.

The goal of this section is to use the above sequence $u_{n, 0}$ (rescaled so that $\left\|u_{n, 0}\right\|_{L^{2}}=$ 1 for all $n$ ) to prove the existence of an $H^{1}$ radial solution $u_{\mathrm{c}}$ to (1.1) with initial data $u_{\mathrm{c}, 0}$ such that $\left\|u_{\mathrm{c}, 0}\right\|_{L^{2}}\left\|\nabla u_{\mathrm{c}, 0}\right\|_{L^{2}}<\|Q\|_{L^{2}}\|\nabla Q\|_{L^{2}}$ and $M\left[u_{\mathrm{c}}\right] E\left[u_{\mathrm{c}}\right]=(M E)_{\mathrm{c}}$ for which $\mathrm{SC}\left(u_{\mathrm{c}, 0}\right)$ does not hold (Proposition 5.4). Moreover, we will show that $K=\left\{u_{\mathrm{c}}(t) \mid 0 \leq t<+\infty\right\}$ is precompact in $H^{1}$ (Proposition [5.5), which will enable us to show that for each $\epsilon>0$, there is an $R>0$ such that, uniformly in $t$, we have

$$
\int_{|x|>R}\left|\nabla u_{\mathrm{c}}(t, x)\right|^{2} d x \leq \epsilon
$$

(Lemma 5.6). This will then play into the rigidity theorem of the next section that will ultimately lead to a contradiction.

Before stating and proving Proposition 5.4, we introduce some preliminaries in the spirit of the results of Keraani [14], since we are not able to directly apply his results as was possible in Kenig-Merle [16]. Note in the following lemma that $\phi_{n}, \psi^{j}$ and $W_{n}^{M}$ are functions of $x$ alone, in notational contrast to the analogous lemma in Keraani ([14] Proposition 2.6).

Lemma 5.2 (Profile expansion). Let $\phi_{n}(x)$ be a radial uniformly bounded sequence in $H^{1}$. Then for each $M$ there exists a subsequence of $\phi_{n}$, also denoted $\phi_{n}$, and

(1) For each $1 \leq j \leq M$, there exists a (fixed in $n$ ) radial profile $\psi^{j}(x)$ in $H^{1}$,

(2) For each $1 \leq j \leq M$, there exists a sequence (in $n$ ) of time shifts $t_{n}^{j}$, 
(3) There exists a sequence (in $n$ ) of remainders $W_{n}^{M}(x)$ in $H^{1}$, such that

$$
\phi_{n}=\sum_{j=1}^{M} e^{-i t_{n}^{j} \Delta} \psi^{j}+W_{n}^{M} .
$$

The time sequences have a pairwise divergence property: For $1 \leq i \neq j \leq M$, we have

$$
\lim _{n \rightarrow+\infty}\left|t_{n}^{i}-t_{n}^{j}\right|=+\infty .
$$

The remainder sequence has the following asymptotic smallness property 6 :

$$
\lim _{M \rightarrow+\infty}\left[\lim _{n \rightarrow+\infty}\left\|e^{i t \Delta} W_{n}^{M}\right\|_{S\left(\dot{H}^{1 / 2}\right)}\right]=0 .
$$

For fixed $M$ and any $0 \leq s \leq 1$, we have the asymptotic Pythagorean expansion

$$
\left\|\phi_{n}\right\|_{\dot{H}^{s}}^{2}=\sum_{j=1}^{M}\left\|\psi^{j}\right\|_{\dot{H}^{s}}^{2}+\left\|W_{n}^{M}\right\|_{\dot{H}^{s}}^{2}+o_{n}(1) .
$$

Note that we do not claim that the remainder $W_{n}^{M}$ is small in any Sobolev norm, i.e. for all we know it might be true that for some $s, 0 \leq s \leq 1$, we have

$$
\liminf _{M \rightarrow+\infty}\left[\lim _{n \rightarrow+\infty}\left\|W_{n}^{M}\right\|_{\dot{H}^{s}}\right]>0 \text {. }
$$

Fortunately, the Strichartz norm smallness (5.2) will suffice in our application.

Proof. Since $\phi_{n}$ is assumed uniformly bounded in $H^{1}$, let $c_{1}$ be such that $\left\|\phi_{n}\right\|_{H^{1}} \leq c_{1}$.

Note the interpolation inequality

$$
\|v\|_{L_{t}^{q} L_{x}^{r}} \leq\|v\|_{L_{t}^{\tilde{q}} L_{x}^{\tilde{r}}}^{1-\theta}\|v\|_{L_{t}^{\infty} L_{x}^{3}}^{\theta},
$$

where $(q, r)$ is any $\dot{H}^{1 / 2}$ Strichartz admissible pair (so $\frac{2}{q}+\frac{3}{r}=1$ and $3 \leq r<+\infty$ ), $\theta=\frac{3}{2 r-3}($ so $0<\theta \leq 1), \tilde{r}=2 r$, and $\tilde{q}=\frac{4 r}{2 r-3}$. Observe that $(\tilde{q}, \tilde{r})$ is also $\dot{H}^{1 / 2}$ Strichartz admissible. By this inequality and the Strichartz estimates (for $0<\theta<\frac{3}{5}$ ) we get

$$
\left\|e^{i t \Delta} W_{n}^{M}\right\|_{L_{t}^{q} L_{x}^{r}} \leq c\left\|W_{n}^{M}\right\|_{\dot{H}^{1 / 2}}^{1-\theta}\left\|e^{i t \Delta} W_{n}^{M}\right\|_{L_{t}^{\infty} L_{x}^{3}}^{\theta} .
$$

Since we will have $\left\|W_{n}^{M}\right\|_{\dot{H}^{1 / 2}} \leq c_{1}$, it will suffice for us to show that

$$
\lim _{M \rightarrow+\infty}\left[\limsup _{n \rightarrow+\infty}\left\|e^{i t \Delta} W_{n}^{M}\right\|_{L_{t}^{\infty} L_{x}^{3}}\right]=0 .
$$

Let $A_{1} \equiv \lim \sup _{n \rightarrow+\infty}\left\|e^{i t \Delta} \phi_{n}\right\|_{L_{t}^{\infty} L_{x}^{3}}$. If $A_{1}=0$, the proof is complete with $\psi^{j}=0$ for all $1 \leq j \leq M$. Suppose $A_{1}>0$. Pass to a subsequence so that

\footnotetext{
${ }^{6}$ We can always pass to a subsequence in $n$ with the property that $\left\|e^{i t \Delta} W_{n}^{M}\right\|_{S\left(\dot{H}^{1 / 2}\right)}$ converges. Therefore, we use lim and not limsup or liminf. Similar remarks apply for the limits that appear in the Pythagorean expansion.

${ }^{7}$ This restriction is for consistency with our definition of $S\left(\dot{H}^{1 / 2}\right)$ in $₫ 2$,
} 
$\lim _{n \rightarrow+\infty}\left\|e^{i t \Delta} \phi_{n}\right\|_{L_{t}^{\infty} L_{x}^{3}}=A_{1}$. We will show that there is a time sequence $t_{n}^{1}$ and a profile $\psi^{1} \in H^{1}$ such that $e^{i t_{n}^{1} \Delta} \phi_{n} \rightarrow \psi^{1}$ and $\left\|\psi^{1}\right\|_{H^{1 / 2}} \geq \frac{A_{1}^{5}}{2^{10} c_{1}^{4}}$. For $r>1$ yet to be chosen, let $\chi(x)$ be a radial Schwartz function such that $\hat{\chi}(\xi)=1$ for $\frac{1}{r} \leq|\xi| \leq r$ and $\hat{\chi}(\xi)$ is supported in $\frac{1}{2 r} \leq|\xi| \leq 2 r$.

By Sobolev embedding,

$$
\begin{aligned}
\left\|e^{i t \Delta} \phi_{n}-\chi * e^{i t \Delta} \phi_{n}\right\|_{L_{t}^{\infty} L_{x}^{3}}^{2} & \leq \int|\xi|(1-\hat{\chi}(\xi))^{2}\left|\hat{\phi}_{n}(\xi)\right|^{2} d \xi \\
& \leq\left.\int_{|\xi| \leq \frac{1}{r}}|\xi \|| \hat{\phi}_{n}(\xi)\right|^{2} d \xi+\int_{|\xi| \geq r}|\xi|\left|\hat{\phi}_{n}(\xi)\right|^{2} d \xi \\
& \leq \frac{1}{r}\left\|\phi_{n}\right\|_{L^{2}}^{2}+\frac{1}{r}\left\|\phi_{n}\right\|_{\dot{H}^{1}}^{2} \\
& \leq \frac{c_{1}^{2}}{r} .
\end{aligned}
$$

Take $r=\frac{16 c_{1}^{2}}{A_{1}^{2}}$ so that $\frac{c_{1}^{2}}{r}=\frac{A_{1}^{2}}{16}$, and then we have for $n$ large

$$
\left\|\chi * e^{i t \Delta} \phi_{n}\right\|_{L_{t}^{\infty} L_{x}^{3}} \geq \frac{1}{2} A_{1} .
$$

Note that

$$
\begin{aligned}
\left\|\chi * e^{i t \Delta} \phi_{n}\right\|_{L_{t}^{\infty} L_{x}^{3}}^{3} & \leq\left\|\chi * e^{i t \Delta} \phi_{n}\right\|_{L_{t}^{\infty} L_{x}^{2}}^{2}\left\|\chi * e^{i t \Delta} \phi_{n}\right\|_{L_{t}^{\infty} L_{x}^{\infty}} \\
& \leq\left\|\phi_{n}\right\|_{L^{2}}^{2}\left\|\chi * e^{i t \Delta} \phi_{n}\right\|_{L_{t}^{\infty} L_{x}^{\infty}},
\end{aligned}
$$

and thus, we have

$$
\left\|\chi * e^{i t \Delta} \phi_{n}\right\|_{L_{t}^{\infty} L_{x}^{\infty}} \geq \frac{A_{1}^{3}}{8 c_{1}^{2}} .
$$

Since $\phi_{n}$ are radial functions, so are $\chi * e^{i t \Delta} \phi_{n}$, and by the radial Gagliardo-Nirenberg inequality, we obtain

$$
\left\|\chi * e^{i t \Delta} \phi_{n}\right\|_{L_{t}^{\infty} L_{\{|x| \geq R\}}^{\infty}} \leq \frac{1}{R}\left\|\chi * e^{i t \Delta} \phi_{n}\right\|_{L_{x}^{2}}^{1 / 2}\left\|\nabla \chi * e^{i t \Delta} \phi_{n}\right\|_{L_{x}^{2}}^{1 / 2} \leq \frac{c_{1}}{R} .
$$

Therefore, by selecting $R$ large enough

$$
\left\|\chi * e^{i t \Delta} \phi_{n}\right\|_{L_{t}^{\infty} L_{\{|x| \leq R\}}^{\infty}} \geq \frac{A_{1}^{3}}{16 c_{1}^{2}} .
$$

Let $t_{n}^{1}$ and $x_{n}^{1}$ (with $\left|x_{n}^{1}\right| \leq R$ ) be sequences such that for each $n$,

$$
\left|\chi * e^{i t_{n}^{1} \Delta} \phi_{n}\left(x_{n}^{1}\right)\right| \geq \frac{A_{1}^{3}}{32 c_{1}^{2}},
$$

or, written out,

$$
\left|\int_{\mathbb{R}^{3}} \chi\left(x_{n}^{1}-y\right) e^{i t_{n}^{1} \Delta} \phi_{n}(y) d y\right| \geq \frac{A_{1}^{3}}{32 c_{1}^{2}} .
$$


Pass to a subsequence such that $x_{n}^{1} \rightarrow x^{1}$ (possible since $\left|x_{n}^{1}\right| \leq R$ ). Then since $\left\|\chi\left(x^{1}-\cdot\right)-\chi\left(x_{n}^{1}-\cdot\right)\right\|_{L^{3 / 2}} \rightarrow 0$ as $n \rightarrow+\infty$, we have

$$
\left|\int_{\mathbb{R}^{3}} \chi\left(x^{1}-y\right) e^{i t_{n}^{1} \Delta} \phi_{n}(y) d y\right| \geq \frac{A_{1}^{3}}{64 c_{1}^{2}} .
$$

Consider the sequence $e^{i t_{n}^{1} \Delta} \phi_{n}$, which is uniformly bounded in $H^{1}$. Pass to a subsequence so that $e^{i t_{n}^{1} \Delta} \phi_{n} \rightarrow \psi^{1}$, with $\psi^{1} \in H^{1}$ radial and $\left\|\psi^{1}\right\|_{H^{1}} \leq \lim \sup \left\|\phi_{n}\right\|_{H^{1}} \leq c_{1}$. By the above, we have

$$
\left|\int_{\mathbb{R}^{3}} \chi\left(x^{1}-y\right) \psi^{1}(y) d y\right| \geq \frac{A_{1}^{3}}{64 c_{1}^{2}} .
$$

By Plancherel and Cauchy-Schwarz applied to the left side of the above inequality, we obtain

$$
\|\chi\|_{\dot{H}^{-1 / 2}}\left\|\psi^{1}\right\|_{\dot{H}^{1 / 2}} \geq \frac{A_{1}^{3}}{64 c_{1}^{2}} .
$$

By converting to radial coordinates, we can estimate $\|\chi\|_{\dot{H}^{-1 / 2}} \leq r$. Thus,

$$
\left\|\psi^{1}\right\|_{\dot{H}^{1 / 2}} \geq \frac{A_{1}^{3}}{64 c_{1}^{2}} \cdot \frac{1}{r}=\frac{A_{1}^{5}}{2^{10} c_{1}^{4}} .
$$

Let $W_{n}^{1}=\phi_{n}-e^{-i t_{n}^{1} \Delta} \psi^{1}$. Since $e^{i t_{n}^{1} \Delta} \phi_{n} \rightarrow \psi^{1}$, we have that for any $0 \leq s \leq 1$

$$
\left\langle\phi_{n}, e^{-i t_{n}^{1} \Delta} \psi^{1}\right\rangle_{\dot{H}^{s}}=\left\langle e^{i t_{n}^{1} \Delta} \phi_{n}, \psi^{1}\right\rangle_{\dot{H}^{s}} \rightarrow\left\|\psi^{1}\right\|_{\dot{H}^{s}}^{2},
$$

and, by expanding $\left\|W_{n}^{1}\right\|_{H^{s}}^{2}$, we obtain

$$
\lim _{n \rightarrow+\infty}\left\|W_{n}^{1}\right\|_{\dot{H}^{s}}^{2}=\lim _{n \rightarrow+\infty}\left\|\phi_{n}\right\|_{\dot{H}^{s}}^{2}-\left\|\psi^{1}\right\|_{\dot{H}^{s}}^{2} .
$$

From this with $s=1$ and $s=0$ we deduce that $\left\|W_{n}^{1}\right\|_{H^{1}} \leq c_{1}$.

Let $A_{2}=\lim \sup _{n \rightarrow+\infty}\left\|e^{i t \Delta} W_{n}^{1}\right\|_{L_{t}^{\infty} L_{x}^{3}}$. If $A_{2}=0$, then we are done. If $A_{2}>0$, then repeat the above argument, with $\phi_{n}$ replaced by $W_{n}^{1}$ to obtain a sequence of time shifts $t_{n}^{2}$ and a profile $\psi^{2} \in H^{1}$ such that $e^{i t_{n}^{2} \Delta} W_{n}^{1} \rightarrow \psi^{2}$ and

$$
\left\|\psi^{2}\right\|_{\dot{H}^{1 / 2}} \geq \frac{A_{2}^{5}}{2^{10} c_{1}^{4}} .
$$

We claim that $\left|t_{n}^{2}-t_{n}^{1}\right| \rightarrow+\infty$. Indeed, suppose we pass to a subsequence such that $t_{n}^{2}-t_{n}^{1} \rightarrow t^{21}$ finite. Then

$$
e^{i\left(t_{n}^{2}-t_{n}^{1}\right) \Delta}\left[e^{i t_{n}^{1} \Delta} \phi_{n}-\psi^{1}\right]=e^{i t_{n}^{2} \Delta}\left[\phi_{n}-e^{-i t_{n}^{1} \Delta} \psi^{1}\right]=e^{i t_{n}^{2} \Delta} W_{n}^{1} \rightarrow \psi^{2} .
$$

Since $t_{n}^{2}-t_{n}^{1} \rightarrow t^{21}$ and $e^{i t_{n}^{1} \Delta} \phi_{n}-\psi^{1} \rightarrow 0$, the left side of the above expression converges weakly to 0 , so $\psi^{2}=0$, a contradiction. Let $W_{n}^{2}=\phi_{n}-e^{i t_{n}^{1} \Delta} \psi^{1}-e^{i t_{n}^{2} \Delta} \psi^{2}$. 
Note that

$$
\begin{aligned}
\left\langle\phi_{n}, e^{i t_{n}^{2} \Delta} \psi^{2}\right\rangle_{\dot{H}^{s}} & =\left\langle e^{-i t_{n}^{2} \Delta} \phi_{n}, \psi^{2}\right\rangle_{\dot{H}^{s}} \\
& =\left\langle e^{-i t_{n}^{2} \Delta}\left(\phi_{n}-e^{i t_{n}^{1} \Delta} \psi^{1}\right), \psi^{2}\right\rangle_{\dot{H}^{s}}+o_{n}(1) \\
& =\left\langle e^{-i t_{n}^{2} \Delta} W_{n}^{1}, \psi^{2}\right\rangle_{\dot{H}^{s}}+o_{n}(1) \\
& \rightarrow\left\|\psi^{2}\right\|_{\dot{H}^{s}}^{2},
\end{aligned}
$$

where the second line follows from the fact that $\left|t_{n}^{1}-t_{n}^{2}\right| \rightarrow \infty$. Using this and (5.4), we compute

$$
\lim _{n \rightarrow+\infty}\left\|W_{n}^{2}\right\|_{\dot{H}^{s}}^{2}=\lim _{n \rightarrow+\infty}\left\|\phi_{n}\right\|_{\dot{H}^{s}}^{2}-\left\|\psi^{1}\right\|_{\dot{H}^{s}}^{2}-\left\|\psi^{2}\right\|_{\dot{H}^{s}}^{2},
$$

and thus, $\left\|W_{n}^{2}\right\|_{H^{1}} \leq c_{1}$.

We continue inductively, constructing a sequence $t_{n}^{M}$ and a profile $\psi^{M}$ such that $e^{i t_{n}^{M} \Delta} W_{n}^{M-1} \rightarrow \psi^{M}$ and

$$
\left\|\psi^{M}\right\|_{\dot{H}^{1 / 2}} \geq \frac{A_{M}^{5}}{2^{10} c_{1}^{4}}
$$

Suppose $1 \leq j<M$. We shall show that $\left|t_{n}^{M}-t_{n}^{j}\right| \rightarrow+\infty$ inductively by assuming that $\left|t_{n}^{M}-t_{n}^{j+1}\right| \rightarrow+\infty, \ldots,\left|t_{n}^{M}-t_{n}^{M-1}\right| \rightarrow+\infty$. Suppose, passing to a subsequence that $t_{n}^{M}-t_{n}^{j} \rightarrow t^{M j}$ finite. Note that

$e^{i\left(t_{n}^{M}-t_{n}^{j}\right) \Delta}\left(e^{i t_{n}^{j} \Delta} W_{n}^{j-1}-\psi^{j}\right)-e^{i\left(t_{n}^{M}-t_{n}^{j+1}\right) \Delta} \psi^{j+1}-\cdots-e^{i\left(t_{n}^{M}-t_{n}^{M-1}\right) \Delta} \psi^{M-1}=e^{i t_{n}^{M} \Delta} W_{n}^{M-1}$.

The left side converges weakly to 0 , while the right side converges weakly to $\psi^{M}$, which is nonzero; contradiction. This proves (5.1). Let $W_{n}^{M}=\phi_{n}-e^{-i t_{n}^{1} \Delta} \psi^{1}-\cdots-$ $e^{-i t_{n}^{M} \Delta} \psi^{M}$. Note that

$$
\begin{aligned}
\left\langle\phi_{n}, e^{-i t_{n}^{M} \Delta} \psi^{M}\right\rangle & =\left\langle e^{i t_{n}^{M} \Delta} \phi_{n}, \psi^{M}\right\rangle_{\dot{H}^{s}} \\
& =\left\langle e^{i t_{n}^{M} \Delta}\left(\phi_{n}-e^{i t_{n}^{1} \Delta} \psi^{1}-\cdots-e^{i t_{n}^{M-1} \Delta} \psi^{M-1}\right), \psi^{M}\right\rangle_{\dot{H}^{s}}+o_{n}(1) \\
& =\left\langle e^{i t_{n}^{M} \Delta} W_{n}^{M-1}, \psi^{M}\right\rangle_{\dot{H}^{s}}+o_{n}(1),
\end{aligned}
$$

where the middle line follows from the pairwise divergence property (5.1). Thus, $\left\langle\phi_{n}, e^{-i t_{n}^{M} \Delta} \psi^{M}\right\rangle \rightarrow\left\|\psi^{M}\right\|_{\dot{H}^{s}}^{2}$. The expansion (5.3) is then shown to hold by expanding $\left\|W_{n}^{M}\right\|_{\dot{H}^{s}}^{2}$.

By (5.5) and (5.3) with $s=\frac{1}{2}$, we have

$$
\sum_{M=1}^{+\infty}\left(\frac{A_{M}^{5}}{2^{10} c_{1}^{4}}\right)^{2} \leq \lim _{n \rightarrow+\infty}\left\|\phi_{n}\right\|_{\dot{H}^{1 / 2}}^{2} \leq c_{1}^{2},
$$

and hence, $A_{M} \rightarrow 0$ as $M \rightarrow+\infty$. 
Corollary 5.3 (Energy Pythagorean expansion). In the situation of Lemma 5.2, we have

$$
E\left[\phi_{n}\right]=\sum_{j=1}^{M} E\left[e^{-i t_{n}^{j} \Delta} \psi^{j}\right]+E\left[W_{n}^{M}\right]+o_{n}(1) .
$$

Proof. We will use the compact embedding $H_{\text {rad }}^{1} \hookrightarrow L_{\text {rad }}^{4}$ (which follows from the radial Gagliardo-Nirenberg estimate of Strauss [25]) to address a $j$ for which $t_{n}^{j}$ converges to a finite number (if one exists). We will also use the decay of linear Schrödinger solutions in the $L^{4}$ norm as time $\rightarrow \infty$.

There are two cases to consider.

Case 1. There exists some $j$ for which $t_{n}^{j}$ converges to a finite number, which without loss we assume is 0 . In this case we will show that

$$
\begin{gathered}
\lim _{n \rightarrow+\infty}\left\|W_{n}^{M}\right\|_{L_{x}^{4}}=0, \quad \text { for } M>j, \\
\lim _{n \rightarrow+\infty}\left\|e^{-i t_{n}^{i} \Delta} \psi^{i}\right\|_{L_{x}^{4}}=0, \quad \text { for all } i \neq j,
\end{gathered}
$$

and

$$
\lim _{n \rightarrow+\infty}\left\|\phi_{n}\right\|_{L^{4}}=\left\|\psi^{j}\right\|_{L^{4}}
$$

which, combined with (5.3) for $s=1$, gives (5.6).

Case 2. For all $j,\left|t_{n}^{j}\right| \rightarrow \infty$. In this case we will show that

$$
\lim _{n \rightarrow+\infty}\left\|e^{-i t_{n}^{j} \Delta} \psi^{j}\right\|_{L_{x}^{4}}=0, \quad \text { for all } j
$$

and

$$
\lim _{n \rightarrow+\infty}\left\|\phi_{n}\right\|_{L^{4}}=\lim _{n \rightarrow+\infty}\left\|W_{n}^{M}\right\|_{L^{4}}
$$

which, combined with (5.3) for $s=1$, gives (5.6).

Proof of Case 1. In this situation, we have, from the proof of Lemma 5.2 that $W_{n}^{j-1} \rightarrow \psi^{j}$. By the compactness of the embedding $H_{\mathrm{rad}}^{1} \hookrightarrow L_{\mathrm{rad}}^{4}$, it follows that $W_{n}^{j-1} \rightarrow \psi^{j}$ strongly in $L^{4}$. Let $i \neq j$. Then we claim that $\left\|e^{i t_{n}^{i} \Delta} \psi^{i}\right\|_{L^{4}} \rightarrow 0$ as $n \rightarrow \infty$. Indeed, since $t_{n}^{j}=0$, by (5.1), we have $\left|t_{n}^{i}\right| \rightarrow+\infty$. For a function $\tilde{\psi}^{i} \in \dot{H}^{3 / 4} \cap L^{4 / 3}$, from Sobolev embedding and the $L^{p}$ spacetime decay estimate of the linear flow, we obtain

$$
\left\|e^{i t_{n}^{j} \Delta} \psi^{i}\right\|_{L^{4}} \leq c\left\|\psi^{i}-\tilde{\psi}^{i}\right\|_{\dot{H}^{3 / 4}}+\frac{c}{\left|t_{n}^{i}\right|^{1 / 4}}\left\|\tilde{\psi}^{i}\right\|_{L^{4 / 3}}
$$

By approximating $\psi^{i}$ by $\tilde{\psi}^{i} \in C_{c}^{\infty}$ in $\dot{H}^{3 / 4}$ and sending $n \rightarrow+\infty$, we obtain the claim. Recalling that

$$
W_{n}^{j-1}=\phi_{n}-e^{-i t_{n}^{1} \Delta} \psi^{1}-\cdots-e^{-i t_{n}^{j-1} \Delta} \psi^{j-1},
$$

we conclude that $\phi_{n} \rightarrow \psi^{j}$ strongly in $L^{4}$. Recalling that

$$
W_{n}^{M}=\left(W_{n}^{j-1}-\psi^{j}\right)-e^{-i t_{n}^{j+1} \Delta} \psi^{j+1}-\cdots-e^{-i t_{n}^{M} \Delta} \psi^{M},
$$


we also conclude that $W_{n}^{M} \rightarrow 0$ strongly in $L^{4}$ for $M>j$.

Proof of Case 2. Similar to the proof of Case 1.

Proposition 5.4 (Existence of a critical solution). There exists a global $\left(T^{*}=+\infty\right)$ solution $u_{\mathrm{c}}$ in $H^{1}$ with initial data $u_{\mathrm{c}, 0}$ such that $\left\|u_{\mathrm{c}, 0}\right\|_{L^{2}}=1$,

$$
\begin{gathered}
E\left[u_{\mathrm{c}}\right]=(M E)_{\mathrm{c}}<M[Q] E[Q], \\
\left\|\nabla u_{\mathrm{c}}(t)\right\|_{L^{2}}<\|Q\|_{L^{2}}\|\nabla Q\|_{L^{2}} \quad \text { for all } 0 \leq t<+\infty
\end{gathered}
$$

and

$$
\left\|u_{\mathrm{c}}\right\|_{S\left(\dot{H}^{1 / 2}\right)}=+\infty
$$

Proof. We consider the sequence $u_{n, 0}$ described in the introduction to this section. Rescale it so that $\left\|u_{n, 0}\right\|_{L^{2}}=1$; this rescaling does not affect the quantities $M\left[u_{n}\right] E\left[u_{n}\right]$ and $\left\|u_{n, 0}\right\|_{L^{2}}\left\|\nabla u_{n, 0}\right\|_{L^{2}}$. After this rescaling, we have $\left\|\nabla u_{n, 0}\right\|_{L^{2}}<\|Q\|_{L^{2}}\|\nabla Q\|_{L^{2}}$ and $E\left[u_{n}\right] \searrow(M E)_{\mathrm{c}}$. Each $u_{n}$ is global and non-scattering, i.e. $\left\|u_{n}\right\|_{S\left(\dot{H}^{1 / 2}\right)}=+\infty$. Apply the profile expansion lemma (Lemma 5.2) to $u_{n, 0}$ (which is now uniformly bounded in $H^{1}$ ) to obtain

$$
u_{n, 0}=\sum_{j=1}^{M} e^{-i t_{n}^{j} \Delta} \psi^{j}+W_{n}^{M}
$$

where $M$ will be taken large later. By the energy Pythagorean expansion (Corollary 5.3), we have

$$
\sum_{j=1}^{M} \lim _{n \rightarrow+\infty} E\left[e^{-i t_{n}^{j} \Delta} \psi^{j}\right]+\lim _{n \rightarrow+\infty} E\left[W_{n}^{M}\right]=\lim _{n \rightarrow+\infty} E\left[u_{n, 0}\right]=(M E)_{\mathrm{c}}
$$

and thus (recalling that each energy is $\geq 0$ - see Lemma 4.5),

$$
\lim _{n \rightarrow+\infty} E\left[e^{-i t_{n}^{j} \Delta} \psi^{j}\right] \leq(M E)_{\mathrm{c}} \quad \forall j
$$

Also by $s=0$ of (5.3), we have

$$
\sum_{j=1}^{M} M\left[\psi^{j}\right]+\lim _{n \rightarrow+\infty} M\left[W_{n}^{M}\right]=\lim _{n \rightarrow+\infty} M\left[u_{n, 0}\right]=1 .
$$

Now we consider two cases; we will show that Case 1 leads to a contradiction and thus does not occur; Case 2 will manufacture the desired critical solution $u_{\mathrm{c}}$.

Case 1. More than one $\psi^{j} \neq 0$. By (5.9), we necessarily have $M\left[\psi^{j}\right]<1$ for each $j$, which by (5.8) implies that for $n$ sufficiently large,

$$
M\left[e^{-i t_{n}^{j} \Delta} \psi^{j}\right] E\left[e^{-i t_{n}^{j} \Delta} \psi^{j}\right]<(M E)_{\mathrm{c}} .
$$


For a given $j$, there are two cases to consider: Case (a). If $\left|t_{n}^{j}\right| \rightarrow+\infty$ (passing to a subsequence we have $t_{n}^{j} \rightarrow+\infty$ or $t_{n}^{j} \rightarrow-\infty$ ) we have $\left\|e^{-i t_{n}^{j} \Delta} \psi^{j}\right\|_{L^{4}} \rightarrow 0$ (as discussed in the proof of Corollary [5.3), and thus,

$$
\frac{1}{2}\left\|\psi^{j}\right\|_{L^{2}}^{2}\left\|\nabla \psi^{j}\right\|_{L^{2}}^{2}<(M E)_{\mathrm{c}}
$$

(we have used $\left\|\nabla e^{-i t_{n}^{j} \Delta} \psi^{j}\right\|_{L^{2}}=\left\|\nabla \psi^{j}\right\|_{L^{2}}$ ). Let $\operatorname{NLS}(t) \psi$ denote the solution to (1.1) with initial data $\psi$. By the existence of wave operators (Proposition 4.6), there exists $\tilde{\psi}^{j}$ such that

$$
\left\|\operatorname{NLS}\left(-t_{n}^{j}\right) \tilde{\psi}^{j}-e^{-i t_{n}^{j} \Delta} \psi^{j}\right\|_{H^{1}} \rightarrow 0, \quad \text { as } n \rightarrow+\infty
$$

with

$$
\begin{gathered}
\left\|\tilde{\psi}^{j}\right\|_{L^{2}}\left\|\nabla \operatorname{NLS}(t) \tilde{\psi}^{j}\right\|_{L^{2}}<\|Q\|_{L^{2}}\|\nabla Q\|_{L^{2}}, \\
M\left[\tilde{\psi}^{j}\right]=\left\|\psi^{j}\right\|_{L^{2}}^{2}, \quad E\left[\tilde{\psi}^{j}\right]=\frac{1}{2}\left\|\nabla \psi^{j}\right\|_{L^{2}}^{2},
\end{gathered}
$$

and thus,

$$
M\left[\tilde{\psi}^{j}\right] E\left[\tilde{\psi}^{j}\right]<(M E)_{\mathrm{c}}, \quad\left\|\mathrm{NLS}(t) \tilde{\psi}^{j}\right\|_{S\left(\dot{H}^{1 / 2}\right)}<+\infty .
$$

Case (b). On the other hand, if for a given $j$ we have $t_{n}^{j} \rightarrow t_{*}$ finite (and there can be at most one such $j$ by (5.1) ), we note that by continuity of the linear flow in $H^{1}$,

$$
e^{-i t_{n}^{j} \Delta} \psi^{j} \rightarrow e^{-i t_{*} \Delta} \psi^{j} \quad \text { strongly in } H^{1}
$$

and we let $\tilde{\psi}^{j}=\operatorname{NLS}\left(t_{*}\right)\left[e^{-i t_{*} \Delta} \psi^{j}\right]$ so that $\operatorname{NLS}\left(-t_{*}\right) \tilde{\psi}^{j}=e^{-i t_{*} \Delta} \psi^{j}$. In either case, associated to each original profile $\psi^{j}$ we now have a new profile $\tilde{\psi}^{j}$ such that

$$
\left\|\operatorname{NLS}\left(-t_{n}^{j}\right) \tilde{\psi}^{j}-e^{-i t_{n}^{j} \Delta} \psi^{j}\right\|_{H^{1}} \rightarrow 0 \quad \text { as } n \rightarrow+\infty \text {. }
$$

It now follows that we can replace $e^{-i t_{n}^{j} \Delta} \psi^{j}$ by $\operatorname{NLS}\left(-t_{n}^{j}\right) \tilde{\psi}^{j}$ in (5.7) to obtain

$$
u_{n, 0}=\sum_{j=1}^{M} \operatorname{NLS}\left(-t_{n}^{j}\right) \tilde{\psi}^{j}+\tilde{W}_{n}^{M},
$$

where

$$
\lim _{M \rightarrow+\infty}\left[\lim _{n \rightarrow+\infty}\left\|e^{i t \Delta} \tilde{W}_{n}^{M}\right\|_{S\left(\dot{H}^{1 / 2}\right)}\right]=0 .
$$

The idea of what follows is that we approximate

$$
\operatorname{NLS}(t) u_{n, 0} \approx \sum_{j=1}^{M} \operatorname{NLS}\left(t-t_{n}^{j}\right) \tilde{\psi}^{j}
$$

via a perturbation theory argument, and since the right side has bounded $S\left(\dot{H}^{1 / 2}\right)$ norm, so must the left-side, which is a contradiction. To carry out this argument, we 
introduce the notation $v^{j}(t)=\operatorname{NLS}(t) \tilde{\psi}^{j}, u_{n}(t)=\operatorname{NLS}(t) u_{n, 0}$, and 8

$$
\tilde{u}_{n}(t)=\sum_{j=1}^{M} v^{j}\left(t-t_{n}^{j}\right) .
$$

Then

$$
i \partial_{t} \tilde{u}_{n}+\Delta \tilde{u}_{n}+\left|\tilde{u}_{n}\right|^{2} \tilde{u}_{n}=e_{n}
$$

where

$$
e_{n}=\left|\tilde{u}_{n}\right|^{2} \tilde{u}_{n}-\sum_{j=1}^{M}\left|v^{j}\left(t-t_{n}^{j}\right)\right|^{2} v^{j}\left(t-t_{n}^{j}\right) .
$$

We claim that there is a (large) constant $A$ (independent of $M$ ) with the property that for any $M$, there exists $n_{0}=n_{0}(M)$ such that for $n>n_{0}$,

$$
\left\|\tilde{u}_{n}\right\|_{S\left(\dot{H}^{1 / 2}\right)} \leq A \text {. }
$$

Moreover, we claim that for each $M$ and $\epsilon>0$ there exists $n_{1}=n_{1}(M, \epsilon)$ such that for $n>n_{1}$,

$$
\left\|e_{n}\right\|_{L_{t}^{10 / 3} L_{x}^{5 / 4}} \leq \epsilon .
$$

Note that since $\tilde{u}_{n}(0)-u_{n}(0)=\tilde{W}_{n}^{M}$, there exists $M_{1}=M_{1}(\epsilon)$ sufficiently large such that for each $M>M_{1}$ there exists $n_{2}=n_{2}(M)$ such that $n>n_{2}$ implies

$$
\left\|e^{i t \Delta}\left(\tilde{u}_{n}(0)-u_{n}(0)\right)\right\|_{S\left(\dot{H}^{1 / 2}\right)} \leq \epsilon .
$$

Thus, we may apply Proposition 2.3 (long-time perturbation theory) to obtain that for $n$ and $M$ sufficiently large, $\left\|u_{n}\right\|_{S\left(\dot{H}^{1 / 2}\right)}<\infty$, a contradiction 9

Therefore, it remains to establish the above claims, and we begin with showing that $\left\|\tilde{u}_{n}\right\|_{S\left(\dot{H}^{1 / 2}\right)} \leq A$ for $n>n_{0}=n_{0}(M)$, where $A$ is some large constant independent of $M$. Let $M_{0}$ be large enough so that

$$
\left\|e^{i t \Delta} \tilde{W}_{n}^{M_{0}}\right\|_{S\left(\dot{H}^{1 / 2}\right)} \leq \delta_{\mathrm{sd}} .
$$

Then for each $j>M_{0}$, we have $\left\|e^{i t \Delta} \psi^{j}\right\|_{S\left(\dot{H}^{1 / 2}\right)} \leq \delta_{\mathrm{sd}}$, and by the second part of Proposition 4.6 we obtain

$$
\left\|v^{j}\right\|_{S\left(\dot{H}^{1 / 2}\right)} \leq 2\left\|e^{i t \Delta} \psi^{j}\right\|_{S\left(\dot{H}^{1 / 2}\right)} \text { for } j>M_{0} .
$$

By the elementary inequality: for $a_{j} \geq 0$,

$$
\left|\left(\sum_{j=1}^{M} a_{j}\right)^{5 / 2}-\sum_{j=1}^{M} a_{j}^{5 / 2}\right| \leq c_{M} \sum_{j \neq k}\left|a_{j}\right|\left|a_{k}\right|^{3 / 2},
$$

\footnotetext{
${ }^{8} \tilde{u}_{n}$, and $e_{n}$ also depend on $M$, but we have suppressed the notation.

${ }^{9}$ The order of logic here is: The constant $A$, which is independent of $M$, is put into Prop. 2.3. which gives a suitable $\epsilon$. We then take $M_{1}=M_{1}(\epsilon)$ as above, and then take $n=\max \left(n_{0}, n_{1}, n_{2}\right)$.
} 
we have

$$
\begin{aligned}
\left\|\tilde{u}_{n}\right\|_{L_{t}^{5} L_{x}^{5}}^{5} & =\sum_{j=1}^{M_{0}}\left\|v^{j}\right\|_{L_{t}^{5} L_{x}^{5}}^{5}+\sum_{j=M_{0}+1}^{M}\left\|v^{j}\right\|_{L_{t}^{5} L_{x}^{5}}^{5}+\text { cross terms } \\
& \leq \sum_{j=1}^{M_{0}}\left\|v^{j}\right\|_{L_{t}^{5} L_{x}^{5}}^{5}+2^{5} \sum_{j=M_{0}+1}^{M}\left\|e^{i t \Delta} \psi^{j}\right\|_{L_{t}^{5} L_{x}^{5}}^{5}+\text { cross terms }
\end{aligned}
$$

where we used (5.10) to bound middle terms. On the other hand, by (5.7),

$$
\left\|e^{i t \Delta} u_{n, 0}\right\|_{L_{t}^{5} L_{x}^{5}}^{5}=\sum_{j=1}^{M_{0}}\left\|e^{i t \Delta} \psi^{j}\right\|_{L_{t}^{5} L_{x}^{5}}^{5}+\sum_{j=M_{0}+1}^{M}\left\|e^{i t \Delta} \psi^{j}\right\|_{L_{t}^{5} L_{x}^{5}}^{5}+\text { cross terms . }
$$

The "cross terms" are made $\leq 1$ by taking $n_{0}=n_{0}(M)$ large enough and appealing to (5.1). We observe that since $\left\|e^{i t \Delta} u_{n, 0}\right\|_{L_{t}^{5} L_{x}^{5}} \leq c\left\|u_{n, 0}\right\|_{\dot{H}^{1 / 2}} \leq c^{\prime}$, (5.12) shows that the quantity $\sum_{j=M_{0}+1}^{M}\left\|e^{i t \Delta} \psi^{j}\right\|_{L_{t}^{5} L_{x}^{5}}^{5}$ is bounded independently of $M$ provided $n>n_{0}$. Then, (5.11) gives that $\left\|\tilde{u}_{n}\right\|_{L_{t}^{5} L_{x}^{5}}$ is bounded independently of $M$ provided $n>n_{0}$. A similar argument establishes that $\left\|\tilde{u}_{n}\right\|_{L_{t}^{\infty} L_{x}^{3}}$ is bounded independently of $M$ for $n>n_{0}$. Interpolation between these exponents gives that $\left\|\tilde{u}_{n}\right\|_{L_{t}^{20} L_{x}^{10 / 3}}$ is bounded independently of $M$ for $n>n_{0}$. Finally, by applying the Kato estimate (2.2) to the integral equation for $i \partial_{t} \tilde{u}_{n}+\Delta \tilde{u}_{n}+\left|\tilde{u}_{n}\right|^{2} \tilde{u}_{n}=e_{n}$ and using that $\left\|e_{n}\right\|_{S\left(\dot{H}^{-1 / 2}\right)} \leq 1$ (proved next), we obtain that $\left\|\tilde{u}_{n}\right\|_{S\left(\dot{H}^{1 / 2}\right)}$ is bounded independently of $M$ for $n>n_{0}$.

We now address the next claim, that for each $M$ and $\epsilon>0$, there exists $n_{1}=$ $n_{1}(M, \epsilon)$ such that for $n>n_{1},\left\|e_{n}\right\|_{L_{t}^{10 / 3} L_{x}^{5 / 4}} \leq \epsilon$. The expansion of $e_{n}$ consists of $\sim M^{3}$ cross terms of the form

$$
v^{j}\left(t-t_{n}^{j}\right) v^{k}\left(t-t_{n}^{k}\right) v^{\ell}\left(t-t_{n}^{\ell}\right),
$$

where not all three of $j, k$, and $\ell$ are the same. Assume, without loss, that $j \neq k$, and thus, $\left|t_{n}^{j}-t_{n}^{k}\right| \rightarrow \infty$ as $n \rightarrow+\infty$. We estimate

$\left\|v^{j}\left(t-t_{n}^{j}\right) v^{k}\left(t-t_{n}^{k}\right) v^{\ell}\left(t-t_{n}^{\ell}\right)\right\|_{L_{t}^{10 / 3} L_{x}^{5 / 4}} \leq\left\|v^{j}\left(t-t_{n}^{j}\right) v^{k}\left(t-t_{n}^{k}\right)\right\|_{L_{t}^{10} L_{x}^{5 / 3}}\left\|v^{\ell}\left(t-t_{n}^{\ell}\right)\right\|_{L_{t}^{5} L_{x}^{5}}$.

Now observe that

$$
\left\|v^{j}\left(t-\left(t_{n}^{j}-t_{n}^{k}\right)\right) \cdot v^{k}(t)\right\|_{L_{t}^{10} L_{x}^{5 / 3}} \rightarrow 0
$$

since $v^{j}$ and $v^{k}$ belong to $L_{t}^{20} L_{x}^{10 / 3}$ and $\left|t_{n}^{j}-t_{n}^{k}\right| \rightarrow \infty$.

Case 2. $\psi^{1} \neq 0$, and $\psi^{j}=0$ for all $j \geq 2$.

By (5.9), we have $M\left[\psi^{1}\right] \leq 1$ and by (5.8), we have $\lim _{n \rightarrow+\infty} E\left[e^{-i t_{n}^{1} \Delta} \psi^{1}\right] \leq(M E)_{\mathrm{c}}$. If $t_{n}^{1}$ converges (to 0 without loss of generality), we take $\tilde{\psi}^{1}=\psi^{1}$ and then we have $\left\|\mathrm{NLS}\left(-t_{n}^{1}\right) \tilde{\psi}^{1}-e^{-i t_{n}^{1} \Delta} \psi^{1}\right\|_{H^{1}} \rightarrow 0$ as $n \rightarrow+\infty$. If, on the other hand, $t_{n}^{1} \rightarrow+\infty$, then since $\left\|e^{i t_{n}^{1} \Delta} \psi^{1}\right\|_{L^{4}} \rightarrow 0$,

$$
\frac{1}{2}\left\|\nabla \psi^{1}\right\|_{L^{2}}^{2}=\lim _{n \rightarrow+\infty} E\left[e^{-i t_{n}^{1} \Delta} \psi^{1}\right] \leq(M E)_{\mathrm{c}}
$$


Thus, by the existence of wave operators (Proposition 4.6), there exists $\tilde{\psi}^{1}$ such that $M\left[\tilde{\psi}^{1}\right]=M\left[\psi^{1}\right] \leq 1, E\left[\tilde{\psi}^{1}\right]=\frac{1}{2}\left\|\nabla \psi^{1}\right\|_{L^{2}}^{2} \leq(M E)_{\mathrm{c}}$, and $\| \mathrm{NLS}\left(-t_{n}^{1}\right) \tilde{\psi}^{1}-$ $e^{-i t_{n}^{1} \Delta} \psi^{1} \|_{H^{1}} \rightarrow 0$ as $n \rightarrow+\infty$.

In either case, let $\tilde{W}_{n}^{M}=W_{n}^{M}+\left(e^{-i t_{n}^{1} \Delta} \psi^{1}-\operatorname{NLS}\left(-t_{n}^{1}\right) \tilde{\psi}^{1}\right)$. Then, by the Strichartz estimates,

$$
\left\|e^{-i t \Delta} \tilde{W}_{n}^{M}\right\|_{S\left(\dot{H}^{1 / 2}\right)} \leq\left\|e^{-i t \Delta} W_{n}^{M}\right\|_{S\left(\dot{H}^{1 / 2}\right)}+c\left\|e^{-i t_{n}^{1} \Delta} \psi^{1}-\operatorname{NLS}\left(-t_{n}^{1}\right) \tilde{\psi}^{1}\right\|_{\dot{H}^{1 / 2}}
$$

and therefore, $\lim _{n \rightarrow+\infty}\left\|e^{-i t \Delta} \tilde{W}_{n}^{M}\right\|_{S\left(\dot{H}^{1 / 2}\right)}=\lim _{n \rightarrow+\infty}\left\|e^{-i t \Delta} W_{n}^{M}\right\|_{S\left(\dot{H}^{1 / 2}\right)}$. Hence, we now have

$$
u_{n, 0}=\operatorname{NLS}\left(-t_{n}^{1}\right) \tilde{\psi}^{1}+\tilde{W}_{n}^{M}
$$

with $M\left[\tilde{\psi}^{1}\right] \leq 1, E\left[\tilde{\psi}^{1}\right] \leq(M E)_{\mathrm{c}}$, and

$$
\limsup _{M \rightarrow+\infty}\left[\lim _{n \rightarrow+\infty}\left\|\tilde{W}_{n}^{M}\right\|_{S\left(\dot{H}^{1 / 2}\right)}\right]=0 \text {. }
$$

Let $u_{\mathrm{c}}$ be the solution to (1.1) with initial data $u_{\mathrm{c}, 0}=\tilde{\psi}^{1}$. Now we claim that $\left\|u_{\mathrm{c}}\right\|_{S\left(\dot{H}^{1 / 2}\right)}=\infty$, and thus, $M\left[u_{\mathrm{c}}\right]=1$ and $E\left[u_{\mathrm{c}}\right]=(M E)_{\mathrm{c}}$, which will complete the proof. To establish this claim, we use a perturbation argument similar to that in Case 1. Suppose

$$
A:=\left\|\mathrm{NLS}\left(t-t_{n}^{1}\right) \tilde{\psi}^{1}\right\|_{S\left(\dot{H}^{1 / 2}\right)}=\left\|\mathrm{NLS}(t) \tilde{\psi}^{1}\right\|_{S\left(\dot{H}^{1 / 2}\right)}=\left\|u_{\mathrm{c}}\right\|_{S\left(\dot{H}^{1 / 2}\right)}<\infty .
$$

Obtain $\epsilon_{0}=\epsilon_{0}(A)$ from the long-time perturbation theory (Proposition 2.3), and then take $M$ sufficiently large and $n_{2}=n_{2}(M)$ sufficiently large so that $n>n_{2}$ implies $\left\|\tilde{W}_{n}^{M}\right\|_{S\left(\dot{H}^{1 / 2}\right)} \leq \epsilon_{0}$. We then repeat the argument in Case 1 using Proposition 2.3 to obtain that there exists $n$ large for which $\left\|u_{n}\right\|_{S\left(\dot{H}^{1 / 2}\right)}<\infty$, a contradiction.

Proposition 5.5 (Precompactness of the flow of the critical solution). With $u_{\mathrm{c}}$ as in Proposition 5.4, let

$$
K=\left\{u_{\mathrm{c}}(t) \mid t \in[0,+\infty)\right\} \subset H^{1} .
$$

Then $K$ is precompact in $H^{1}$ (i.e. $\bar{K}$ is compact in $H^{1}$ ).

Proof. Take a sequence $t_{n} \rightarrow+\infty$; we shall argue that $u_{\mathrm{c}}\left(t_{n}\right)$ has a subsequence converging in $H^{1} 10$ Take $\phi_{n}=u_{\mathrm{c}}\left(t_{n}\right)$ (a uniformly bounded sequence in $H^{1}$ ) in the profile expansion lemma (Lemma 5.2) to obtain profiles $\psi^{j}$ and an error $W_{n}^{M}$ such that

$$
u_{\mathrm{c}}\left(t_{n}\right)=\sum_{j=1}^{M} e^{-i t_{n}^{j} \Delta} \psi^{j}+W_{n}^{M}
$$

\footnotetext{
${ }^{10} \mathrm{By}$ time continuity of the solution in $H^{1}$, we of course do not need to consider the case when $t_{n}$ is bounded and thus has a subsequence convergent to some finite time.
} 
with $\left|t_{n}^{j}-t_{n}^{k}\right| \rightarrow+\infty$ as $n \rightarrow+\infty$ for fixed $j \neq k$. By the energy Pythagorean expansion (Corollary 5.3), we have

$$
\sum_{j=1}^{M} \lim _{n \rightarrow+\infty} E\left[e^{-i t_{n}^{j} \Delta} \psi^{j}\right]+\lim _{n \rightarrow+\infty} E\left[W_{n}^{M}\right]=E\left[u_{\mathrm{c}}\right]=(M E)_{\mathrm{c}}
$$

and thus (recalling that each energy is $\geq 0-$ see Lemma 4.5),

$$
\lim _{n \rightarrow+\infty} E\left[e^{-i t_{n}^{j} \Delta} \psi^{j}\right] \leq(M E)_{\mathrm{c}} \quad \forall j .
$$

Also by $s=0$ of (5.3), we have

$$
\sum_{j=1}^{M} M\left[\psi^{j}\right]+\lim _{n \rightarrow+\infty} M\left[W_{n}^{M}\right]=\lim _{n \rightarrow+\infty} M\left[u_{n, 0}\right]=1 .
$$

We now consider two cases, just as in the proof of Proposition 5.4, both Case 1 and Case 2 will lead to a contradiction.

Case 1. More than one $\psi^{j} \neq 0$. The proof that this leads to a contradiction is identical to that in Proposition 5.4, so we omit it.

Case 2. Only $\psi^{1} \neq 0$ and $\psi^{j}=0$ for all $2 \leq j \leq M$, so that

$$
u_{\mathrm{c}}\left(t_{n}\right)=e^{-i t_{n}^{1} \Delta} \psi^{1}+W_{n}^{M}
$$

Just as in the proof of Proposition 5.4 Case 2, we obtain that

$$
\begin{gathered}
M\left[\psi^{1}\right]=1, \quad \lim _{n \rightarrow+\infty} E\left[e^{-i t_{n}^{1} \Delta} \psi^{1}\right]=(M E)_{\mathrm{c}}, \\
\lim _{n \rightarrow+\infty} M\left[W_{n}^{M}\right]=0, \text { and } \lim _{n \rightarrow+\infty} E\left[W_{n}^{M}\right]=0 .
\end{gathered}
$$

By the comparability lemma (Lemma 4.5),

$$
\lim _{n \rightarrow+\infty}\left\|W_{n}^{M}\right\|_{H^{1}}=0
$$

Next, we show that (a subsequence of) $t_{n}^{1}$ converges 11 Suppose that $t_{n}^{1} \rightarrow-\infty$. Then

$$
\left\|e^{i t \Delta} u_{\mathrm{c}}\left(t_{n}\right)\right\|_{S\left(\dot{H}^{1 / 2} ;[0,+\infty)\right)} \leq\left\|e^{i\left(t-t_{n}^{1}\right) \Delta} \psi^{1}\right\|_{S\left(\dot{H}^{1 / 2} ;[0,+\infty)\right)}+\left\|e^{i t \Delta} W_{n}^{M}\right\|_{S\left(\dot{H}^{1 / 2} ;[0,+\infty)\right)} .
$$

Since

$$
\lim _{n \rightarrow+\infty}\left\|e^{i\left(t-t_{n}^{1}\right) \Delta} \psi^{1}\right\|_{S\left(\dot{H}^{1 / 2} ;[0,+\infty)\right)}=\lim _{n \rightarrow+\infty}\left\|e^{i t \Delta} \psi^{1}\right\|_{S\left(\dot{H}^{1 / 2} ;\left[-t_{n}^{1},+\infty\right)\right)}=0
$$

and $\left\|e^{i t \Delta} W_{n}^{M}\right\|_{S\left(\dot{H}^{1 / 2}\right)} \leq \frac{1}{2} \delta_{\text {sd }}$, we obtain a contradiction to the small data scattering theory (Proposition 2.1) by taking $n$ sufficiently large. On the other hand, suppose that $t_{n}^{1} \rightarrow+\infty$. Then we can similarly argue that for $n$ large,

$$
\left\|e^{i t \Delta} u_{\mathrm{c}}\left(t_{n}\right)\right\|_{S\left(\dot{H}^{1 / 2} ;(-\infty, 0]\right)} \leq \frac{1}{2} \delta_{\mathrm{sd}}
$$

\footnotetext{
${ }^{11}$ In the rest of the argument, take care not to confuse $t_{n}^{1}$ (associated with $\psi^{1}$ ) with $t_{n}$.
} 
and thus, the small data scattering theory (Proposition 2.1) shows that

$$
\left\|u_{\mathrm{c}}\right\|_{S\left(\dot{H}^{1 / 2} ;\left(-\infty, t_{n}\right]\right)} \leq \delta_{\mathrm{sd}}
$$

Since $t_{n} \rightarrow+\infty$, by sending $n \rightarrow+\infty$ in the above, we obtain $\left\|u_{\mathrm{c}}\right\|_{S\left(\dot{H}^{1 / 2} ;(-\infty,+\infty)\right)} \leq$ $\delta_{\text {sd }}$, a contradiction. Thus, we have shown that $t_{n}^{1}$ converges to some finite $t^{1}$.

Since $e^{-i t_{n}^{1} \Delta} \psi^{1} \rightarrow e^{-i t^{1} \Delta} \psi^{1}$ in $H^{1}$ and (5.14) holds, (5.13) shows that $u_{\mathrm{c}}\left(t_{n}\right)$ converges in $H^{1}$.

Lemma 5.6 (Precompactness of the flow implies uniform localization). Let $u$ be $a$ solution to (1.1) such that

$$
K=\{u(t) \mid t \in[0,+\infty)\}
$$

is precompact in $H^{1}$. Then for each $\epsilon>0$, there exists $R>0$ so that

$$
\int_{|x|>R}|\nabla u(x, t)|^{2} \leq \epsilon, \quad \text { for all } 0 \leq t<+\infty .
$$

Proof. If not, then there exists $\epsilon>0$ and a sequence of times $t_{n}$ such that

$$
\int_{|x|>n}\left|\nabla u\left(x, t_{n}\right)\right|^{2} d x \geq \epsilon .
$$

Since $K$ is precompact, there exists $\phi \in H^{1}$ such that, passing to a subsequence of $t_{n}$, we have $u\left(t_{n}\right) \rightarrow \phi$ in $H^{1}$. By taking $n$ large, we have both

$$
\int_{|x|>n}|\nabla \phi(x)|^{2} \leq \frac{1}{4} \epsilon
$$

and

which is a contradiction.

$$
\int_{\mathbb{R}^{3}}\left|\nabla\left(u\left(x, t_{n}\right)-\phi(x)\right)\right|^{2} d x \leq \frac{1}{4} \epsilon,
$$

\section{Rigidity THEOREM}

We now prove the rigidity theorem.

Theorem 6.1 (Rigidity). Suppose $u_{0} \in H^{1}$ satisfies

$$
M\left[u_{0}\right] E\left[u_{0}\right]<M[Q] E[Q]
$$

and

$$
\left\|u_{0}\right\|_{L^{2}}\left\|\nabla u_{0}\right\|_{L^{2}}<\|Q\|_{L^{2}}\|\nabla Q\|_{L^{2}}
$$

Let $u$ be the global $H^{1}$ solution of (1.1) with initial data $u_{0}$ and suppose that

$$
K=\{u(t) \mid t \in[0,+\infty)\} \quad \text { is precompact in } H^{1} .
$$

Then $u_{0}=0$. 
Proof. Let $\phi \in C_{0}^{\infty}$, radial, with

$$
\phi(x)=\left\{\begin{array}{ll}
|x|^{2} & \text { for }|x| \leq 1 \\
0 & \text { for } \quad|x| \geq 2
\end{array} .\right.
$$

For $R>0$ define $z_{R}(t)=\int R^{2} \phi\left(\frac{x}{R}\right)|u(x, t)|^{2} d x$. Then

$$
\left|z_{R}^{\prime}(t)\right| \leq 2 R\left|\int \bar{u}(t) \nabla u(t)(\nabla \phi)\left(\frac{x}{R}\right) d x\right| \leq c R \int_{0<|x|<2 R}|\nabla u(t)||u(t)| d x .
$$

Using Hölder's inequality and Theorem 1.1(1)(a), we bound the previous expression by

$$
c R\|\nabla u(t)\|_{L^{2}}\|u\|_{L^{2}} \leq c R\|\nabla Q\|_{L^{2}}\|Q\|_{L^{2}}=\tilde{c} R .
$$

Thus, we obtain

$$
\left|z_{R}^{\prime}(t)-z_{R}^{\prime}(0)\right| \leq 2 \tilde{c} R \text { for } \quad t>0
$$

Next we estimate $z_{R}^{\prime \prime}(t)$ using the localized virial identity (4.1):

$$
\begin{aligned}
z_{R}^{\prime \prime}(t)= & 4 \int \phi^{\prime \prime}\left(\frac{|x|}{R}\right)|\nabla u|^{2}-\frac{1}{R^{2}} \int\left(\Delta^{2} \phi\right)\left(\frac{x}{R}\right)|u|^{2}-\int(\Delta \phi)\left(\frac{x}{R}\right)|u|^{4} \\
\geq & 8 \int_{|x| \leq R}|\nabla u|^{2}+4 \int_{R<|x|<2 R} \phi^{\prime \prime}\left(\frac{|x|}{R}\right)|\nabla u|^{2}-\frac{c}{R^{2}} \int_{R<|x|<2 R}|u|^{2} \\
& -6 \int_{|x| \leq R}|u|^{4}-c \int_{R<|x|<2 R}|u|^{4} \\
\geq & \left(8 \int_{|x| \leq R}|\nabla u|^{2}-6 \int_{|x| \leq R}|u|^{4}\right)-c_{1} \int_{R<|x|<2 R}\left(|\nabla u|^{2}+\frac{|u|^{2}}{R^{2}}+|u|^{4}\right) .
\end{aligned}
$$

Since (6.1) holds, take $\delta>0$ such that $M\left[u_{0}\right] E\left[u_{0}\right] \leq(1-\delta) M[Q] E[Q]$. Let $\epsilon=c_{1}^{-1} c_{\delta} \int\left|\nabla u_{0}\right|^{2}$, where $c_{\delta}$ is as in (4.9).

Since $\{u(t) \mid t \in[0, \infty)\}$ is precompact in $H^{1}$, by Lemma 5.6 there exists $R_{1}>0$ such that $\int_{|x|>R_{1}}|\nabla u(t)|^{2} \leq \frac{1}{9} \epsilon$. Next, because of mass conservation, there exists $R_{2}>0$ such that $\frac{1}{R_{2}^{2}} \int|u|^{2}<\frac{1}{9} \epsilon$. Finally, the radial Gagliardo-Nirenberg inequality (3.5) yields the existence of $R_{3}>0$ such that

$$
\int_{|x|>R_{3}}|u(t)|^{4} \leq \frac{c}{R_{3}^{2}}\|\nabla u(t)\|_{L^{2}\left(|x|>R_{3}\right)}\left\|u_{0}\right\|_{L^{2}}^{3} \leq \frac{c}{R_{3}^{2}}\left\|\nabla u_{0}\right\|_{L^{2}}\left\|u_{0}\right\|_{L^{2}}^{3} \leq \frac{1}{9} \epsilon,
$$

with $R_{3}^{2}>9 c \epsilon\left\|\nabla u_{0}\right\|_{L^{2}}\left\|u_{0}\right\|_{L^{2}}^{3}$; in the above chain we used the gradient-energy comparability (Lemma 4.5) with $t=0$ on the left side. Take $R=\max \left\{R_{1}, R_{2}, R_{3}\right\}$ to obtain

$$
c_{1} \int_{|x|>R}\left(|\nabla u|^{2}+\frac{|u|^{2}}{R^{2}}+|u|^{4}\right) \leq \frac{1}{3} c_{\delta} \int\left|\nabla u_{0}\right|^{2} .
$$


By (4.9) and Lemma 4.5, we also have

$$
8 \int|\nabla u|^{2}-6 \int|u|^{4} \geq c_{\delta} \int\left|\nabla u_{0}\right|^{2}
$$

Splitting the integrals on the left side of the above expression into the regions $\{|x|>$ $R\}$ and $\{|x|<R\}$ and applying ( $(\underline{6.5})$, we get

$$
8 \int_{|x| \leq R}|\nabla u|^{2}-6 \int_{|x| \leq R}|u|^{4} \geq \frac{2}{3} c_{\delta} \int\left|\nabla u_{0}\right|^{2} .
$$

Hence, we obtain $z_{R}^{\prime \prime}(t) \geq \frac{1}{3} c_{\delta}\left\|\nabla u_{0}\right\|_{L^{2}}^{2}$, which implies by integration from 0 to $t$ that $z_{R}^{\prime}(t)-z_{R}^{\prime}(0) \geq \frac{1}{3} c_{\delta}\left\|\nabla u_{0}\right\|_{L^{2}}^{2} t$. Taking $t$ large, we obtain a contradiction with (6.4), which can be resolved only if $\left\|\nabla u_{0}\right\|_{L^{2}}=0$.

To complete the proof of Theorem 1.1(1)(b), we just apply Theorem 6.1 to $u_{\mathrm{c}}$ constructed in Proposition 5.4, which by Proposition 5.5, meets the hypotheses in Theorem 6.1. Thus $u_{\mathrm{c}, 0}=0$, which contradicts the fact that $\left\|u_{\mathrm{c}}\right\|_{S\left(\dot{H}^{1 / 2}\right)}=\infty$. We have thus obtained that if $\left\|u_{0}\right\|_{L^{2}}\left\|\nabla u_{0}\right\|_{L^{2}}<\|Q\|_{L^{2}}\|\nabla Q\|_{L^{2}}$ and $M[u] E[u]<$ $M[Q] E[Q]$, then $\mathrm{SC}\left(u_{0}\right)$ holds, i.e. $\|u\|_{S\left(\dot{H}^{1 / 2}\right)}<\infty$. By Proposition $2.2, H^{1}$ scattering holds.

\section{EXTENSIONS TO GENERAL MASS SUPERCRITICAL, ENERGY SUBCRITICAL NLS EQUATIONS}

Consider the focusing mass supercritical, energy subcritical nonlinear Schrödinger equation $\operatorname{NLS}_{p}\left(\mathbb{R}^{N}\right)$ :

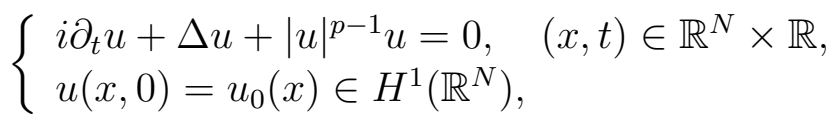

with the choice of nonlinear exponent $p$ and the dimension $N$ such that

$$
0<s_{c}<1, \quad \text { where } \quad s_{c}=\frac{N}{2}-\frac{2}{p-1} .
$$

The initial value problem with $u_{0} \in H^{1}\left(\mathbb{R}^{N}\right)$ is locally well-posed, see [9]. Denote by $I=\left(-T_{*}, T^{*}\right)$ the maximal interval of existence of the solution $u$ (e.g., see [3]). This implies that either $T^{*}=+\infty$ or $T^{*}<+\infty$ and $\|\nabla u(t)\|_{L^{2}} \rightarrow \infty$ as $t \rightarrow T^{*}$ (similar properties for $T_{*}$ ).

The solutions to this problem satisfy mass and energy conservation laws, in particular,

$$
E[u(t)]=\frac{1}{2} \int|\nabla u(x, t)|^{2}-\frac{1}{p+1} \int|u(x, t)|^{p+1} d x=E\left[u_{0}\right] .
$$

The Sobolev $\dot{H}^{s_{c}}$ norm is invariant under the scaling $u \mapsto u_{\lambda}(x, t)=\lambda^{2 /(p-1)} u\left(\lambda x, \lambda^{2} t\right)$ $\left(u_{\lambda}\right.$ is a solution of $\operatorname{NLS}_{p}\left(\mathbb{R}^{N}\right)$, if $u$ is $)$. 
The general Gagliardo-Nirenberg inequality (see [31]) is valid for values of $p$ and $N$ such that $0 \leq s_{c}<12$ :

$$
\|u\|_{L^{p+1}\left(\mathbb{R}^{N}\right)}^{p+1} \leq c_{\mathrm{GN}}\|\nabla u\|_{L^{2}\left(\mathbb{R}^{N}\right)}^{\frac{N(p-1)}{2}}\|u\|_{L^{2}\left(\mathbb{R}^{N}\right)^{2}}^{2-(N-2)(p-1)}
$$

where

$$
c_{\mathrm{GN}}=\frac{\|Q\|_{L^{p+1}\left(\mathbb{R}^{N}\right)}^{p+1}}{\|\nabla Q\|_{L^{2}\left(\mathbb{R}^{N}\right)}^{\frac{N(p-1)}{2}}\|Q\|_{L^{2}\left(\mathbb{R}^{N}\right)}^{2-\frac{(N-2)(p-1)}{2}}}
$$

and $Q$ is the ground state solution (positive solution of minimal $L^{2}$ norm) of the equation

$$
-\left(1-s_{c}\right) Q+\Delta Q+|Q|^{p-1} Q=0 .
$$

(See [31] and references therein for discussion on the existence of positive solutions of class $H^{1}\left(\mathbb{R}^{N}\right)$ to this equation. $\sqrt{13}$ The corresponding soliton solution to (7.1) is $u(x, t)=e^{i\left(1-s_{c}\right) t} Q(x)$.

The generalization of Theorem 4.2 (or Theorem 1.1 without scattering) to all $0<$ $s_{c}<1$ is based on using the scaling invariant quantity $\|\nabla u\|_{L^{2}\left(\mathbb{R}^{N}\right)}^{s_{c}} \cdot\|u\|_{L^{2}\left(\mathbb{R}^{N}\right)}^{1-s_{c}}$.

Theorem 7.1. Consider $N L S_{p}\left(\mathbb{R}^{N}\right)$ with (possibly non-radial) $u_{0} \in H^{1}\left(\mathbb{R}^{N}\right.$ ) and $0<s_{c}<1$. Suppose that

$$
E\left[u_{0}\right]^{s_{c}} M\left[u_{0}\right]^{1-s_{c}}<E[Q]^{s_{c}} M[Q]^{1-s_{c}}, \quad E\left[u_{0}\right] \geq 0 .
$$

If (17.4) holds and

$$
\left\|\nabla u_{0}\right\|_{L^{2}\left(\mathbb{R}^{N}\right)}^{s_{c}}\left\|u_{0}\right\|_{L^{2}\left(\mathbb{R}^{N}\right)}^{1-s_{c}}<\|\nabla Q\|_{L^{2}\left(\mathbb{R}^{N}\right)}^{s_{c}}\|Q\|_{L^{2}\left(\mathbb{R}^{N}\right)}^{1-s_{c}},
$$

then for any $t \in I$,

$$
\|\nabla u(t)\|_{L^{2}\left(\mathbb{R}^{N}\right)}^{s_{c}}\left\|u_{0}\right\|_{L^{2}\left(\mathbb{R}^{N}\right)}^{1-s_{c}}<\|\nabla Q\|_{L^{2}\left(\mathbb{R}^{N}\right)}^{s_{c}}\|Q\|_{L^{2}\left(\mathbb{R}^{N}\right)}^{1-s_{c}}
$$

and thus $I=(-\infty,+\infty)$, i.e. the solution exists globally in time.

If (7.4) holds and

$$
\left\|\nabla u_{0}\right\|_{L^{2}\left(\mathbb{R}^{N}\right)}^{s_{c}}\left\|u_{0}\right\|_{L^{2}\left(\mathbb{R}^{N}\right)}^{1-s_{c}}>\|\nabla Q\|_{L^{2}\left(\mathbb{R}^{N}\right)}^{s_{c}}\|Q\|_{L^{2}\left(\mathbb{R}^{N}\right)}^{1-s_{c}}
$$

then for $t \in I$

$$
\|\nabla u(t)\|_{L^{2}\left(\mathbb{R}^{N}\right)}^{s_{c}}\left\|u_{0}\right\|_{L^{2}\left(\mathbb{R}^{N}\right)}^{1-s_{c}}>\|\nabla Q\|_{L^{2}\left(\mathbb{R}^{N}\right)}^{s_{c}}\|Q\|_{L^{2}\left(\mathbb{R}^{N}\right)}^{1-s_{c}}
$$

Furthermore, if (a) $|x| u_{0} \in L^{2}\left(\mathbb{R}^{N}\right)$, or (b) $u_{0}$ is radial with $N>1$ and $1+\frac{4}{N}<p<$ $\min \left\{1+\frac{4}{N-2}, 5\right\}$, then $I$ is finite, and thus, the solution blows up in finite time. The

\footnotetext{
${ }^{12}$ It is also valid for $s_{c}=1$ becoming the Sobolev embedding, see Remark 7.3 .

${ }^{13}$ In the case $p=3, N=3$, we have $s_{c}=\frac{1}{2}$, and thus, the normalization for $Q$ chosen here is different from that in the main part of this paper. The normalization of $Q$ taken here was chosen since it enables us to draw a comparison with the $s_{c}=1$ endpoint result of Kenig-Merle [16].
} 
finite-time blowup conclusion and (7.8) also hold if, in place of (7.4) and (17.7), we assume $E\left[u_{0}\right]<0$.

The proof of this theorem is similar to Theorem 4.2 and can be found in [12].

Remark 7.2. A finite-time $T$ blow-up solution to a mass-supercritical energy subcritical NLS equation satisfies a lower bound on the blow-up rate: $\|\nabla u(t)\|_{L^{2}} \geq c(T-t)^{-\alpha}$, where $\alpha=\alpha(p, d)$. This is obtained by scaling the local-theory, and it implies that the quantity $\left\|u_{0}\right\|_{L^{2}}^{s_{c}}\|\nabla u(t)\|_{L^{2}}^{1-s_{c}} \rightarrow \infty$, thus strengthening the conclusion (7.8). A stronger result in this direction was recently obtained by Merle-Raphaël [21]: if $u(t)$ blows-up in finite time $T^{*}<\infty$, then $\lim _{t \rightarrow T^{*}}\|u(t)\|_{\dot{H}^{s_{c}}}=\infty$ (in fact, it diverges to $\infty$ with a logarithmic lower bound).

Remark 7.3. This theorem provides a link between the mass critical NLS and energy critical NLS equations: Consider $s_{c}=1$; the theorem holds true by the work of KenigMerle [16, Section 3]. In this case there is no mass involved, the Gagliardo-Nirenberg inequality (7.2) becomes the Sobolev inequality, the condition (7.4) is $E\left[u_{0}\right]<E[Q]$, where $Q$ is the radial positive decreasing (class $\dot{H}^{1}\left(\mathbb{R}^{N}\right)$ ) solution of $\triangle Q+|Q|^{p-1} Q=$ 0 , and the conditions (7.5) - (7.8) involve only the size of $\left\|\nabla u_{0}\right\|_{L^{2}}$ in relation to $\|\nabla Q\|_{L^{2}\left(\mathbb{R}^{N}\right)}$. In regard to the case $s_{c}=0$, (17.4) should be replaced by $M[u]<M[Q]$ and (17.5) becomes the same statement. Under this hypotheses, the result of $\mathrm{M}$. Weinstein [31] states that

$$
\|\nabla u(t)\|_{L^{2}}^{2} \leq 2\left(1-\frac{\left\|u_{0}\right\|_{L^{2}}^{2}}{\|Q\|_{L^{2}}^{2}}\right)^{-1} E[u], \quad E[u]>0,
$$

and thus, global existence holds. We do not recover this estimate as a formal limit in (17.6) 14 however, the conclusion about the global existence in this case does hold true. Our intention here is not to reprove the $s_{c}=0$ endpoint result - only to draw a connection to it. The hypothesis (17.7) should be replaced by its formal limit $\left\|u_{0}\right\|_{L^{2}}>\|Q\|_{L^{2}}$, which is the complement of (7.4). Thus, the only surviving claim in Theorem 7.1 regarding blow-up in the $s_{c}=0$ limit is that it should hold under the hypothesis $E\left[u_{0}\right]<0$. Blow-up under this hypothesis is the classical result of Glassey [11] in the case of finite variance, and in the radial case it is the result of Ogawa-Tsutsumi [22].

We expect that the proof of scattering for $\operatorname{NLS}_{p}\left(\mathbb{R}^{N}\right)$ with $u_{0} \in H^{1}\left(\mathbb{R}^{N}\right)$ and $0<s_{c}<1$ when (7.4) and (7.5) hold will carry over analogously to the $N=3, p=3$ case, provided (i) $N>1$ (the radial assumption in 1D does not help to eliminate the translation defect of compactness); (ii) the Kato estimate (as in (2.2) ) or the more

\footnotetext{
${ }^{14}$ It might appear as a formal limit if one were to refine the estimate (7.6) to account for the gain resulting from the strict inequality in (7.4) (as we did in the proof of Theorem 4.2) before passing to the $s_{c} \rightarrow 0$ limit.
} 
refined Strichartz estimates by Foschi [8] are sufficient to complete the long term perturbation argument 15 .

\section{REFERENCES}

[1] L. Bergé, T. Alexander, and Y. Kivshar, Stability criterion for attractive Bose-Einstein condensates, Phys. Rev. A, 62 (2000).

[2] P. Bégout, Necessary conditions and sufficient conditions for global existence in the nonlinear Schrödinger equation, Adv. Math. Sci. Appl. 12 (2002), no. 2, pp. 817-827.

[3] T. Cazenave, Semilinear Schrödinger equations. Courant Lecture Notes in Mathematics, 10. New York University, Courant Institute of Mathematical Sciences, New York; American Mathematical Society, Providence, RI, 2003. xiv+323 pp. ISBN: 0-8218-3399-5.

[4] J. Colliander, M. Keel, G. Staffilani, H. Takaoka, T. Tao, Global existence and scattering for rough solutions of a nonlinear Schrödinger equation on $\mathbb{R}^{3}$, Comm. Pure Appl. Math. 57 (2004), no. 8, pp. 987-1014.

[5] J. Colliander, M. Keel, G. Staffilani, H. Takaoka, T. Tao, Global well-posedness and scattering for the energy-critical nonlinear Schrödinger equation in $R^{3}$, arxiv.org preprint arXiv:math/0402129v7 [math.AP].

[6] E. Donley, N. Claussen, S. Cornish, J. Roberts, E. Cornell, and C. Wieman, Dynamics of collapsing and exploding Bose-Einstein condensates, Nature 412 (2001) pp. 295.

[7] G. Fibich, Some modern aspects of self-focusing theory, in Self-Focusing: Past and Present, R.W. Boyd, S.G. Lukishova, Y.R. Shen, editors, to be published by Springer. Available at http://www.math.tau.ac.il/ fibich/publications.html.

[8] D. Foschi, Inhomogeneous Strichartz estimates, J. Hyper. Diff. Eq. 2 (2005), no. 1, 1-24.

[9] J. Ginibre and G. Velo, On a class of nonlinear Schrödinger equation. I. The Cauchy problems; II. Scattering theory, general case, J. Func. Anal. 32 (1979), 1-32, pp. 33-71.

[10] J. Ginibre and G. Velo, Scattering theory in the energy space for a class of nonlinear Schrödinger equations, J. Math. Pures Appl. (9) 64 (1985), no. 4, pp. 363-401.

[11] R. T. Glassey, On the blowing up of solutions to the Cauchy problem for nonlinear Schrödinger equation, J. Math. Phys., 18, 1977, 9, pp. 1794-1797.

[12] J. Holmer and S. Roudenko, On blow-up solutions to the $3 D$ cubic nonlinear Schrödinger equation, AMRX Appl. Math. Res. Express, vol. 2007, article ID abm004, doi:10.1093/amrx/abm004.

[13] T. Kato, An $L^{q, r}$-theory for nonlinear Schrödinger equations, Spectral and scattering theory and applications, pp. 223-238, Adv. Stud. Pure Math., 23, Math. Soc. Japan, Tokyo, 1994.

[14] S. Keraani, On the defect of compactness for the Strichartz estimates of the Schrödinger equation, J. Diff. Eq. 175 (2001), pp. 353-392

[15] M. Keel and T. Tao, Endpoint Strichartz estimates, Amer. J. Math., 120 (1998), pp. 955-980.

[16] C.E. Kenig and F. Merle, Global well-posedness, scattering, and blow-up for the energy-critical focusing nonlinear Schrödinger equation in the radial case, Invent. Math. 166 (2006), no. 3, pp. 645-675.

[17] C. Kenig, G. Ponce and L. Vega, Well-posedness and scattering results for the generalized Korteweg-de Vries equation via the contraction principle, Comm. Pure Appl. Math. 46 (1993), no. $4,527-620$.

\footnotetext{
${ }^{15}$ It may be necessary, for example, to express the estimates in terms of the norm $\left\|D^{s_{c}-\alpha}(\cdots)\right\|_{S\left(\dot{H}^{\alpha}\right)}$ for some $0<\alpha<s_{c}$, rather than $\|\cdot\|_{S\left(\dot{H}^{s_{c}}\right)}$.
} 
[18] N.E. Kosmatova, V.F. Shvets and V.E. Zakharov, Computer simulation of wave collapses in the nonlinear Schrödinger equation, Physica D 52 (1991), pp. 16-35.

[19] E.A. Kuznetsov, J. Juul Rasmussen, K. Rypdal, S. K. Turitsyn, Sharper criteria for the wave collapse, Phys. D 87 (1995), no. 1-4, pp. 273-284.

[20] F. Linares, G. Ponce, Introduction to nonlinear dispersive equations, Rio de Janeiro: IMPA, 2004, 243 pp.

[21] F. Merle, P. Raphaël, Blow-up of the critical norm for some radial $L^{2}$ supercritical nonlinear Schrödinger equations, arxiv.org preprint arXiv:math/0605378v2 [math.AP].

[22] T. Ogawa and Y. Tsutsumi, Blow-Up of $H^{1}$ solution for the Nonlinear Schrödinger Equation, J. Diff. Eq. 92 (1991), pp. 317-330.

[23] Schlein, B. Derivation of the Gross-Pitaevskii hierarchy, Mathematical physics of quantum mechanics, pp. 279-293, Lecture Notes in Phys., 690, Springer, Berlin, 2006. See also arxiv.org arXiv:math-ph/0504078.

[24] A. Soffer, Soliton dynamics and scattering, Proceedings of the International Congress of Mathematicians, Madrid, Spain 2006.

[25] W.A. Strauss, Existence of solitary waves in higher dimensions, Comm. Math. Phys. 55 (1977), no. 2, pp. 149-162.

[26] C. Sulem, P-L. Sulem, The nonlinear Schrödinger equation. Self-focusing and wave collapse, Applied Mathematical Sciences, 139. Springer-Verlag, New York, 1999. xvi+350 pp.

[27] T. Tao, On the asymptotic behavior of large radial data for a focusing non-linear Schrödinger equation, Dyn. Partial Differ. Equ. 1 (2004), no. 1, pp. 1-48.

[28] T. Tao, A (concentration-) compact attractor for high-dimensional non-linear Schrödinger equations, Dynamics of PDE 4 (2007) pp. 1-53.

[29] T. Tao, Nonlinear dispersive equations. Local and global analysis. CBMS Regional Conference Series in Mathematics, 106. Published for the Conference Board of the Mathematical Sciences, Washington, DC; by the American Mathematical Society, Providence, RI, 2006. xvi+373 pp. ISBN: 0-8218-4143-2

[30] M. Vilela, Regularity of solutions to the free Schrödinger equation with radial initial data, Illinois J. Math. 45 (2001), no. 2, pp. 361-370.

[31] M. Weinstein, Nonlinear Schrödinger equations and sharp interpolation estimates, Comm. Math. Phys. 87 (1982/83), no. 4, pp. 567-576.

[32] V. E. Zakharov, Collapse of Langmuir waves, Zh. Eksp. Teor. Fiz. 62, (1972), pp. 1745-1751, (in Russian); Sov. Phys. JETP, 35 (1972), pp. 908-914 (English).

University of CALifornia, Berkeley

Arizona State University 